\title{
mTORC2 Drives ZFX-mediated Ganglioside Biosynthesis to Promote Breast Cancer Progression.
}

Kajal Rajput, ${ }^{1, a}$ Mohd. Nafees Ansari, ${ }^{1, a}$ Somesh K. Jha, ${ }^{2}$ Nihal Medatwal, ${ }^{2, b}$ Pankaj Sharma, ${ }^{1}$ Sudeshna Datta, ${ }^{3, c}$ Animesh Kar, ${ }^{2}$ Trishna Pani, ${ }^{1}$ Kaushavi Cholke ${ }^{1}$, Kajal Rana, ${ }^{2}$ Ali Khan, ${ }^{1}$ Geetashree Mukherjee, ${ }^{4}$ SVS Deo, ${ }^{5}$ Jyothi S Prabhu, ${ }^{6}$ Arnab Mukhopadhyay, ${ }^{3}$ Avinash Bajaj, ${ }_{1}^{2, *}$ and Ujjaini Dasgupta ${ }^{1, *}$

1. Amity Institute of Integrative Sciences and Health, Amity University Haryana, Panchgaon, Manesar, Gurgaon-122413, Haryana, India.

2. Regional Centre for Biotechnology, NCR Biotech Science Cluster, $3^{\text {rd }}$ Milestone Faridabad-Gurgaon Expressway, Faridabad-121001, Haryana, India.

3. National Institute of Immunology, Aruna Asaf Ali Marg, New Delhi-110067, India.

4. Tata Medical Center, 14 Main Arterial Road (EW), New Town, Rajarhat, Kolkata-700160, India.

5. Department of Surgical Oncology, BRA-Institute Rotary Cancer Hospital, All India Institute of Medical Sciences, New Delhi-110029, India.

6. Division of Molecular Medicine, St. Johns Research Institute, St Johns National Academy of Health Sciences, Bangalore-560034, India.

a: These authors contributed equally to the work.

b: Current address: Department of Systems Pharmacology and Translational Therapeutics, University of Pennsylvania, Philadelphia, PA, USA

c: Current address: Sudeshna Datta, Data analyst, Institute of Life Sciences, Nalco square, Bhubaneswar751023, India.

\section{Corresponding authors:}

Email: bajaj@rcb.res.in

Email: udasgupta@ggn.amity.edu 


\section{ABSTRACT}

Sphingolipid and ganglioside metabolic pathways are crucial components of cell signalling, having established roles in tumor cell proliferation, invasion, and migration. However, regulatory mechanisms controlling sphingolipid and ganglioside synthesis in mammalian cells is less known. Here, we show that RICTOR, the regulatory subunit of mTORC2, regulates the synthesis of sphingolipids and gangliosides in Luminal breast cancer-specific MCF-7 cells through transcriptional and epigenetic mechanisms. RICTOR regulates glucosylceramide levels by modulating the expression of UDP-Glucose Ceramide Glucosyl transferase (UGCG). We identify Zinc Finger protein X-linked (ZFX) as a RICTORresponsive transcription factor whose recruitment to the UGCG promoter is regulated by DNA methyltransferases and histone demethylase (KDM5A) that are known AKT substrates. We further demonstrate that RICTOR regulates the synthesis of GD3 gangliosides through ZFX and UGCG, and triggers the activation of the EGFR signalling pathway, thereby promoting tumor growth. In line with our findings in cell culture and mice models, we observe an elevated expression of RICTOR, ZFX, and UGCG in Indian Luminal breast cancer patient samples, and in TCGA and METABRIC datasets. Together, we establish a key regulatory circuit, RICTOR-AKT-ZFX-UGCG-Ganglioside-EGFR-AKT, and elucidate its contribution to breast cancer progression. 


\section{INTRODUCTION}

Sphingolipids and gangliosides are metabolically interconnected structural and signalling components of cell membranes, and deregulation in their metabolism is linked to key human diseases including cancer $(1,2)$. Ceramides are the central hub of the sphingolipid pathway, and modifications at their $3^{\prime}-$ hydroxyl terminal lead to structurally diverse classes of sphingolipids like glucosylceramides, sphingomyelins, and ceramide-1-phosphates with a distinct role in different facets of tumorigenesis (Figure 1A) (3). Synthesis of glucosylceramides from ceramides is catalysed by UDP-Glucose Ceramide Glucosyl transferase (UGCG), and ceramide-glucosylceramide rheostat connecting sphingolipid and ganglioside metabolism plays a crucial role in tumor progression, drug resistance, and chemotherapeutic response (Figure 1A) ( ). Gangliosides are sialic acid-containing glycosphingolipids residing in the glycolipid-enriched microdomains (GEMs) of plasma membrane (4). Gangliosides, as residents of GEMs, can induce activation/deactivation of Receptor-Tyrosine Kinases (RTKs), thereby regulating the downstream signalling processes (5). Therefore, understanding the mechanisms that regulate the ceramide-glucosylceramide rheostat connecting sphingolipid and ganglioside pathways, and its impact on RTK signalling may potentially lead to identification of new therapeutic targets.

$\mathrm{PI3K} / \mathrm{AKT} / \mathrm{mTOR}$ is the key downstream pathway of most RTKs (6), and is hyperactivated in $60 \%$ of breast cancer patients due to increased expression of growth factors and their receptors or genetic alterations in PIK3CA, AKT, and PTEN ( ). Mammalian-target of Rapamycin (mTOR), an intracellular serine/threonine kinase of the PI3K pathway exists in two different complexes, mTORC1 and mTORC2. Rapamycin-Insensitive Companion of mTOR (RICTOR) is the key regulatory subunit of mTORC2 that can directly phosphorylate AKT at $\operatorname{Ser}^{473}$ (8). Apart from AKT activation, mTORC2 also regulates tumorigenesis through activation of other substrates like AGC kinases, Serum- and Glucocorticoidinduced protein kinase 1 (SGK), and Protein Kinase C (PKC) (9-12). Amplification of R/CTOR or elevated RICTOR protein expression is positively correlated with poor overall survival of cancer patients (13-17). Meta-analysis of cancer genomics datasets show co-occurrence of RTK alterations with RICTOR overexpression in different cancer types (18). Therefore, there is a need to unravel the role of RICTOR (mTORC2)-mediated regulatory pathways in tumorigenesis. 
Metabolic reporgramming is one of the hallmarks of tumorigenesis, and mTORC2 has emerged as a key link between RTK signalling and cancer metabolic reprogramming (19). mTORC2 can regulate the glycolytic metabolism in cancer cells either through AKT phosphorylation, or via regulating the c-Myc expression, or through transcription factor, FoxO (19). mTORC2 also regulates lipogenesis, lipid homeostasis, and adipogenesis in non-cancer cells, however, mTORC2-mediated regulation of sphingolipid and ganglioside in cancer cells is not well understood (20). Yeast studies have shown that TORC2-dependent protein kinase, Ypk1, activates phosphorylation of subunits of ceramide synthase complex, leading to an increased synthesis of sphingolipids (21). It is also known that membrane stress in S. cerevisiae causes redistribution of SIm proteins in plasma membrane, leading to their association with TORC2 complex, and further activation of sphingolipid synthesis (22). Recent studies have shown that mTORC2 may promote tumorigenesis in hepatocarcinoma by promoting de novo fatty acid and lipid synthesis (23). However, mTORC2-mediated regulation of sphingolipid and ganglioside metabolism through transcriptional and epigenetic regulatory mechanisms, and impact of the network surrounding this metabolic hub on tumorigenesis is not known. Herein, we delineate the mTORC2mediated regulation of ceramide-glucosylceramide rheostat, and its role in breast cancer progression.

Luminal $(A$ and $B)$ is the most common breast cancer subtype, accounting for $\sim 75 \%$ of all cases $(24,25)$. Although Luminal breast cancer patients, characterized by the presence of estrogen and progesterone receptor (ER and PR), have a better prognosis, but recurrence and resistance to endocrine therapy are major challenges (25). Here, we show that RICTOR-mediated epigenomic alterations in DNA and histone methylation lead to rewiring of the UGCG transcriptional competence in Luminal breast cancer (MCF-7) cells. We identify ZFX as the RICTOR-responsive transcription factor that regulates UGCG expression. We further demonstrate that elevated expression of ZFX/UGCG alters the ganglioside levels, and activate epidermal growth factor receptor (EGFR)-mediated PI3K/AKT/mTOR/MAPK signalling leading to tumor progression. Finally, Indian patient tumor tissues with high RICTOR expression also manifest high UGCG and ZFX expression, emphasizing the role of mTORC2-regulated ganglioside metabolism in tumor development. 


\section{RESULTS}

\section{Luminal Tumors have High Glucosylceramide Levels and High RICTOR Expression.}

Ceramide-glucosylceramide rheostat plays a crucial role in tumor progression, drug resistance, and chemotherapeutic response where synthesis of glucosylceramides from ceramides is catalysed by UGCG, and glucosylceramidase (GBA) hydrolyses glucosylceramides to ceramides (Figure 1A, B). To detect any imbalance in the ceramide-glucosylceramide rheostat in Luminal breast cancer patients, we quantified the levels of ceramides and glucosylceramides in Luminal patient tumor tissues from an Indian cohort (Dataset 1), and compared them with adjacent normal tissues using liquidchromatography mass spectrometry (LC-MS/MS) in multiple reaction monitoring (MRM) mode $(26,27)$. Tumor tissues showed 1.6-28 fold increase in levels of all ceramide species as compared to their adjacent normal tissues (Figure 1C). Similarly, we observed a 1.8-100 fold increase in levels of all glucosylceramide species in tumor tissues (Figure 1D). Glucosylceramide to ceramide ratio for each species is 1.7-42 fold higher in tumor tissues than in normal tissues suggesting that the rheostat is shifted towards glucosylceramides in Luminal breast cancer patients (Figure 1E).

To decipher the effect of increased glucosylceramides on tumor progression, we overexpressed UGCG transcript-encoding CDNA in Luminal breast cancer representing MCF-7 cells (MCF-7_UGCG ${ }^{\mathrm{OE}}$ ), and used only vector-transfected cells (MCF-7_VECT ${ }^{\mathrm{OE}}$ ) as control. We also silenced UGCG expression by siRNA (MCF-7_UGCG ${ }^{\mathrm{SL}}$ ), and used scrambled siRNA-transfected cells as control (MCF-7_SCRAM ${ }^{\mathrm{SL}}$ ). Overexpression and downregulation of UGCG were confirmed by qRT-PCR (Supplementary Figure S1A), immunoblot (Supplementary Figure S1B), and immunofluorescence staining (Supplementary Figure S1C). As expected, overexpression of UGCG attenuated the levels of ceramides (Supplementary Figure S1D), and MCF-7_UGCGOE cells showed a 4-12 fold increase in levels of all glucosylceramide species (Supplementary Figure S1E) that was also validated by immunostaining of ceramides (Supplementary Figure S1F). MCF-7_UGCGOE cells exhibited elevated cell proliferation (Supplementary Figure S1G) and cell migration (Supplementary Figure $\mathrm{S} 1 \mathrm{H}$ ) as compared to MCF-7_VECT ${ }^{\mathrm{OE}}$ cells. In contrast, UGCG silencing showed a $>1.7$-fold $(p<0.001)$ decrease in cell proliferation (Supplementary Figure S1I), and $>2$-fold decrease $(p<0.001)$ in number of migrating cells (Supplementary Figure S1J) as compared to MCF-7_SCRAM ${ }^{S L}$ cells. Mice xenograft studies recorded a significant increase in tumor growth kinetics 
of MCF-7_UGCGOE tumors (Supplementary Figure S1K) with tumor sections showing high expression of Ki67 (Supplementary Figure S1L) and UGCG (Supplementary Figure S1M) along with low levels of ceramides (Supplementary Figure S1N).

We then investigated the effect of UGCG overexpression on RTK signaling pathway to decipher the mechanism of UGCG-mediated enhanced cell proliferation. Immunoblot studies revealed enhanced expression of RICTOR, pAKT ${ }^{\mathrm{S} 473}$, and $\mathrm{pSGK}^{\mathrm{S} 78}$ in MCF-7_UGCG $\mathrm{OE}$ cells (Supplementary Figure S10), whereas there was no significant change in RAPTOR and mTORC1 targets p4EBP1 and p70S6K (Supplementary Figure S10). We also generated R/CTOR silenced MCF-7_RICTOR ${ }^{\text {SH }}$ cells using lentiviralmediated shRNA transfection (as a control) that showed reduced expression of $\mathrm{pAKT}^{\mathrm{S} 473}$ and $\mathrm{pSGK}^{\mathrm{S78}}$. The activation of PI3K/AKT/mTOR pathway on UGCG expression hinted at a complex feed forward loop involving sphingolipid metabolism, RTKs, and PI3K/AKT/mTOR signaling.

RICTOR being a key component of mTORC2, earlier studies on analysis of The Cancer Genome Atlas (TCGA) curated invasive breast carcinoma patient datasets have shown significant correlation of R/CTOR upregulation, mutation, or amplification with low overall survival (28-30). To validate any deregulation of RICTOR expression in Luminal breast tumors, we quantitated the RICTOR expression by immunofluorescence in tumor and adjoining matched normal breast tissues from Luminal patients from the same Indian cohort (Figure 1F, 1G, Dataset 1). We observed an abundant ( $\mathrm{n}=7, p=0.053)$ increase in RICTOR expression in tumor tissues as compared to adjoining normal tissues (Figure 1G). Above results show that Luminal breast cancer tissues have high levels of glucosylceramides and high RICTOR expression. Therefore, we hypothesized that RICTOR (mTORC2) might regulate the sphingolipid metabolism, and increased levels of glucosylceramides may be instrumental for enhanced cancer cell proliferation and tumor progression in Luminal breast cancer patients (Figure 1H). 
bioRxiv preprint doi: https://doi.org/10.1101/2022.01.10.475595; this version posted January 12, 2022. The copyright holder for this

Figure 1

Luminal tumors have high levels of glucosylceramides.

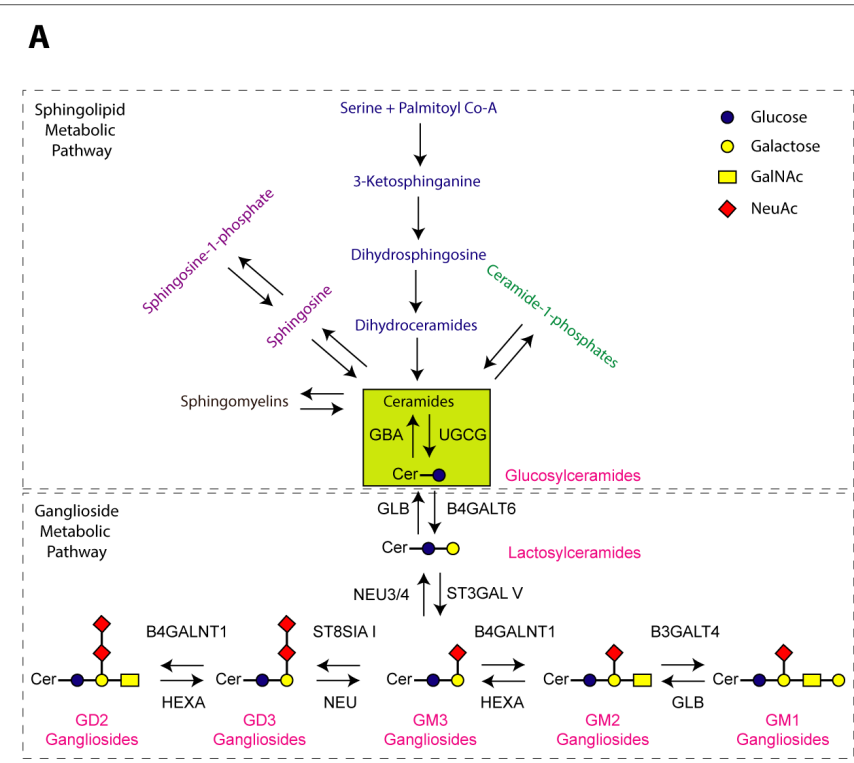

D

B

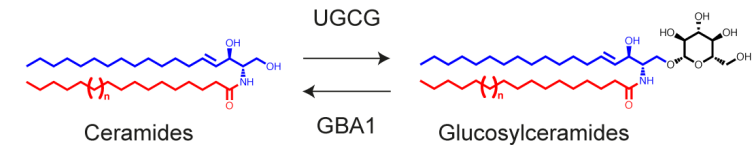

C16:0: $n=1, C 18: 0: n=3, C 20: 0: n=5, C 22: 0: n=7, C 24: 0: n=9, C 24: 1: n=9,-2 H$

C

Ceramides in adjacent normal $(\mathrm{N})$ and tumor $(\mathrm{T})$ tissues

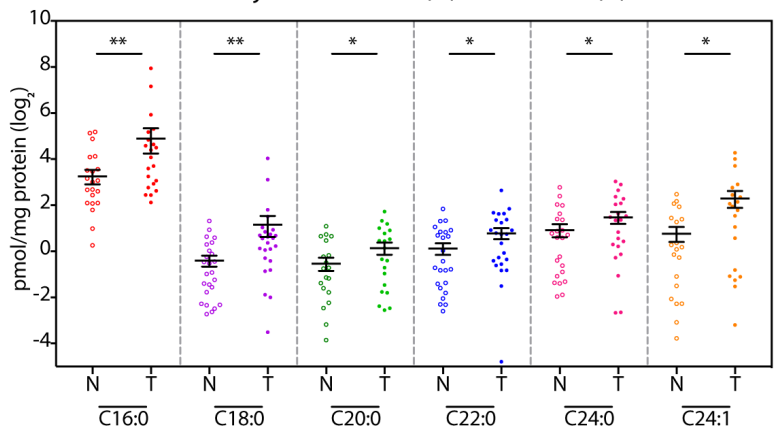

$\mathbf{E}$

Glucosylceramide/ceramide ratio ( $\mathrm{N}=$ normal, $\mathrm{T}=$ tumor $)$

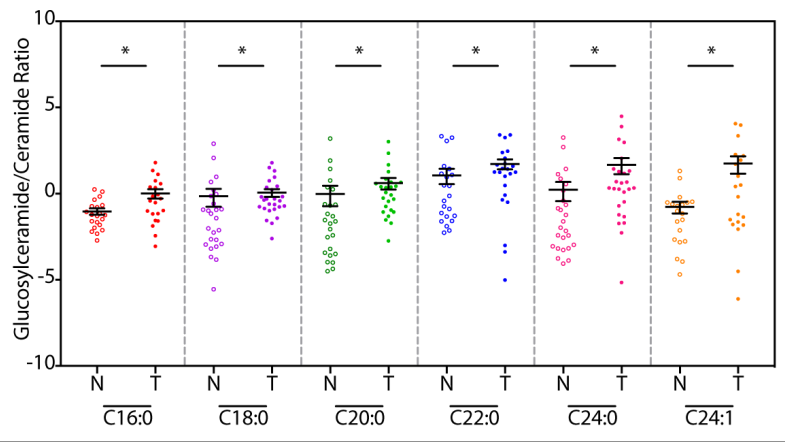

Luminal tumors have high RICTOR expression.

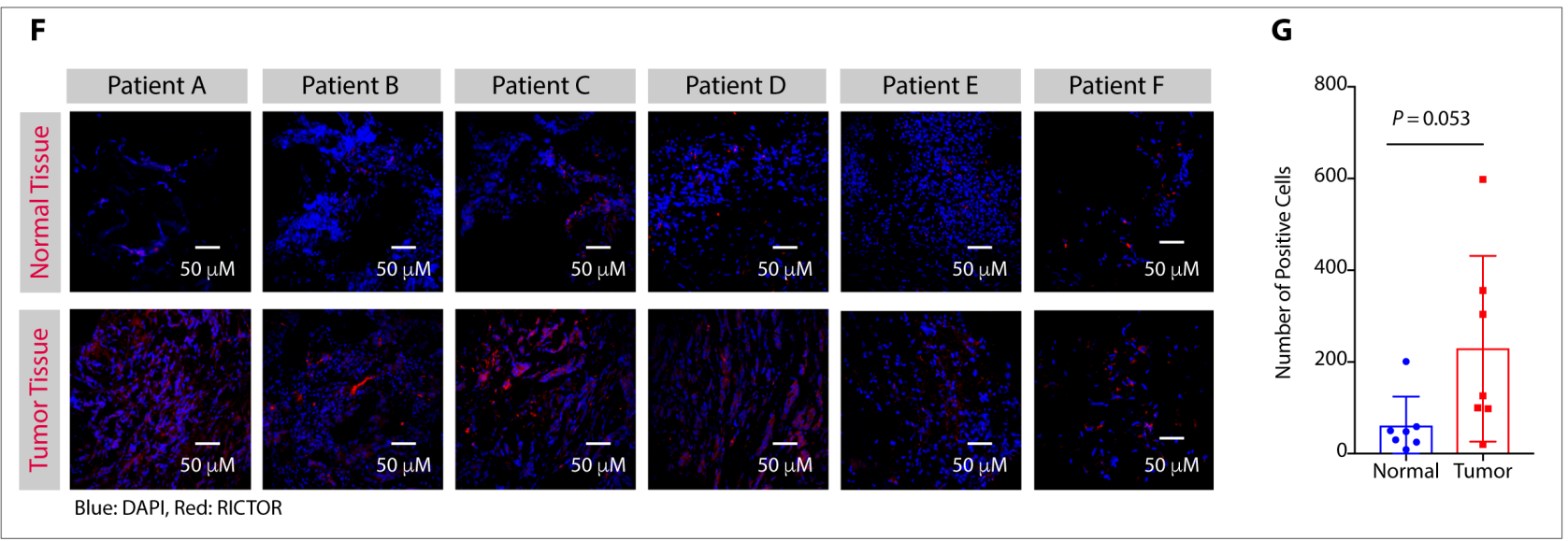

H

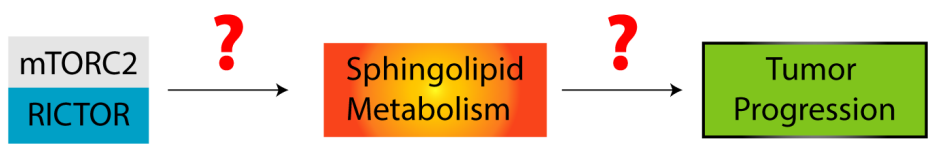


Figure 1. Luminal Tumors have Elevated Glucosylceramide Levels and High RICTOR Expression.

(A) A schematic presentation showing sphingolipid and ganglioside metabolic pathways along with ceramide-glucosylceramide rheostat connecting these pathways. (B) A schematic showing the synthesis of glucosylceramides, with different fatty acyl chain lengths, from ceramides that is catalysed by UGCG, and breakdown of glucosylceramides to ceramides by GBA1. (C, D) Absolute quantitation (pmol/mg protein) of different species (C16:0, C18:0, C20:0, C22:0, C24:0, and C24:1) of ceramides (C) and glucosylceramides (D) show higher levels in Luminal tumor tissues (labelled as T) than adjoining normal tissues (labelled as N). (E) Glucosylceramide to ceramide ratios for Luminal tumor tissues (lablled as T) and adjacent normal tissues (lablled as $\mathrm{N}$ ) indicate that the balance is shifted towards the synthesis of glucosylceramides. $(F, G)$ Immunofluorescence images $(F)$ and quantification $(G)($ mean $\pm S D, n=7$ ) show the elevated RICTOR expression in tumor tissues as compared to adjacent normal tissue sections. (H) A schematic diagram showing questions that needed to be unraveled in order to understand the mTORC2-mediated regulation of sphingolipid-metabolic pathway and their role in tumor progression. Data in Figure 1C, 1D, 1E, and $1 \mathrm{G}$ were analysed using paired student's $t$-test. $p$-value: * $p<0.05,{ }^{* *} p$ $<0.01,{ }^{* * *} p<0.001$. 


\section{RICTOR Silencing Inhibits Cell Proliferation and Migration.}

As Luminal tumor tissues show high RICTOR expression, we first determined the effect of RICTOR silencing on cancer cell proliferation, migration, and invasion in breast cancer Luminal subtype representative MCF-7 cells. We used the engineered RICTOR silenced MCF-7_RICTOR ${ }^{\text {SH }}$ cells and MCF7_SCRAM ${ }^{\mathrm{SH}}$ cells with scrambled shRNA as control (Figure 2A, Supplementary Figure S2A). Cellular assay showed $\sim 1.25$-fold $\left(p<0.0001\right.$ ) decrease in proliferation of MCF-7_RICTOR ${ }^{\text {SH }}$ cells after $72 \mathrm{~h}$ (Figure $2 \mathrm{~B})$, and $>2.0$-fold $(p<0.0001)$ decrease in number of colonies during anchorage-dependent assay as compared to MCF-7_SCRAM ${ }^{\text {SH }}$ cells (Supplementary Figure S2B). Similarly, scratch wound and transwell migration assays showed $>1.3$-fold ( $p<0.0001$ ) (Supplementary Figure S2C), and $>1.5$-fold $(p<0.001)$ (Supplementary Figure S2D) decrease in number of migrating cells on RICTOR silencing. The effect of RICTOR silencing in MCF-7 cells was further validated by quantifying the expression of downstream effectors of mTORC2 pathway including AGC kinases like AKT and SGK. As expected, there was a $\sim 2$-fold decrease in the expression of $\operatorname{pAKT}^{\mathrm{S} 473}(p=0.09)$ and $\operatorname{pSGK}^{\mathrm{S78}}(p<0.05)$ upon RICTOR silencing, and there was no change in expression of mTORC1-specific RAPTOR, p4EBP1 ${ }^{\text {T37 }}$, and p70S6K (Figure 2C, 2D). These experiments suggest that pathogenic activation of RICTOR seen in Luminal cancer tissues may activate proliferation through activation of downstream effectors like AGC kinases. Next, we ventured to understand how RICTOR activation may lead to enhanced proliferation, particularly in the context of sphingolipid metabolism.

\section{mTORC2 Alters Ceramide-Glucosylceramide Rheostat via Regulating UGCG.}

To decipher the effect of RICTOR silencing on sphingolipid metabolism, we performed quantitative profiling of sphingolipids in MCF-7 and MCF-7_RICTOR ${ }^{\text {SH }}$ cells by LC-MS/MS (Figure 2E-2G). All species (C16:0, C18:0, C20:0, C22:0, C24:0, and C24:1) of glucosylceramides showed a significant decrease in MCF-7_RICTOR ${ }^{\text {SH }}$ cells with a concurrent increase in levels of ceramides as compared to MCF-7 cells

(Figure 2E). There is a 2-9 fold increase in levels of ceramide species, and 1.3-1.7 fold decrease in levels of glucosylceramides in MCF-7_RICTOR ${ }^{\mathrm{SH}}$ cells over MCF-7 cells (Figure 2F, G). Immunofluorescent staining with anti-ceramide antibody elicited an increase in levels of total ceramides in MCF-7_ RICTOR $^{S H}$ cells (Figure $2 \mathrm{H}$ ). As UGCG is responsible for the synthesis of glucosylceramides from ceramides, we observed a 2 -fold $(p<0.05)$ decrease in UGCG expression by qRT-PCR in MCF-7_ 
RICTOR $^{\text {SH }}$ cells as compared to MCF-7 cells (Figure 2l) that was also validated by immunoblotting (Figure 2J, 2K), and immunofluorescence imaging (Figure 2L). In contrast, we did not observe any change in expression of GBA1 that hydrolyses glucosylceramides to ceramides (Figure 2J, 2K). qRT-PCR (Supplementary Figure S2E) and immunoblot (Supplementary Figure S2F) analysis showed significant deregulation in expression of other sphingolipid-metabolic genes including ceramide synthases, highlighting the RICTOR-mediated regulation of sphingolipid metabolism. Therefore, these results suggest that increase in levels of ceramides upon RICTOR silencing may be either through downregulation of UGCG or through upregulation of ceramide synthases. As we observed a significant decrease in levels of glucosylceramides and UGCG on RICTOR silencing, we pursued the RICTORmediated regulation of UGCG for further studies.

\section{RICTOR Silencing Inhibits Tumor Progression via UGCG.}

To validate the RICTOR-mediated regulation of UGCG and its impact on tumor progression, we compared tumor growth kinetics of MCF-7_RICTOR ${ }^{S H}$ and MCF-7_SCRAM ${ }^{S H}$ cells in NOD SCID mice, and observed a $>3$-fold $(p<0.001)$ decrease in kinetics of tumor growth following implantation of MCF-7_RICTOR ${ }^{\mathrm{SH}}$ cells as compared to MCF-7_SCRAM ${ }^{\mathrm{SH}}$ cells (Figure 2M-O). Immunostaining of tumor sections showed low Ki67 (proliferation marker) (Figure 2P), low UGCG expression (Supplementary Figure S2G), and high levels of ceramides (Supplementary Figure S2H) in MCF-7_RICTOR ${ }^{\text {SH }}$ tumors as compared to MCF-7_SCRAM ${ }^{\mathrm{SH}}$ tumors. These results helped us to speculate that RICTOR regulates the expression of UGCG at transcriptional level, and increase in glucosylceramides may enhance cell proliferation and tumor progression (Figure 2Q). We next asked how RICTOR regulates the UGCG expression, and how UGCG-mediated glucosylceramide synthesis enhances tumor progression (Figure 2Q). 
Figure 2

RICTOR silencing inhibits cell proliferation in MCF-7 cells.
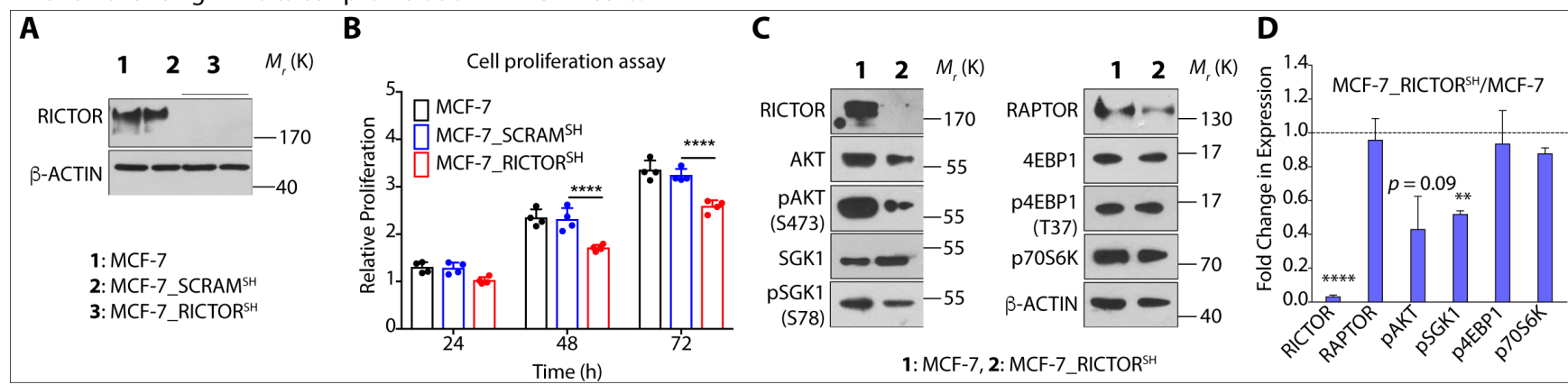

RICTOR silencing increases ceramides and decreases glucosylceramides in MCF-7 cells.
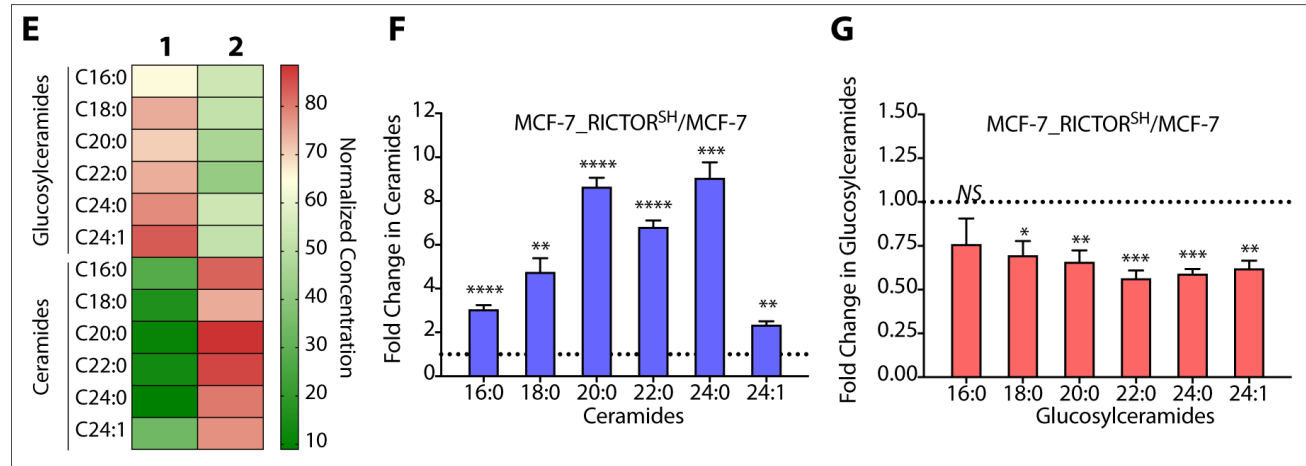

\section{H}

1: MCF-7, 2: MCF-7_RICTOR ${ }^{\text {SH }}$

RICTOR silencing decreases UGCG expression in MCF-7 cells.

I J K

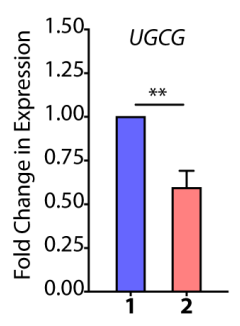

1: MCF-7

2: MCF-7_RICTOR ${ }^{\text {SH }}$

RICTOR silencing slows down tumor progression via regulating UGCG.

$\mathbf{M}$

\section{$\mathbf{N}$}

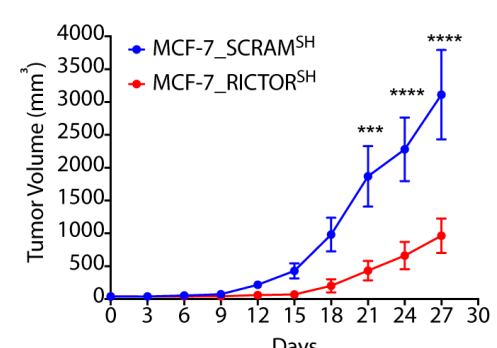

Days
1: MCF-7

2: MCF-7_RICTOR ${ }^{\text {SH }}$

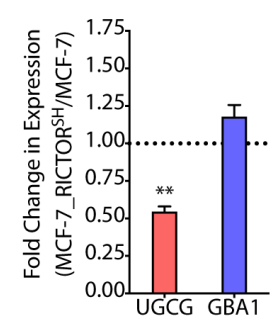

L

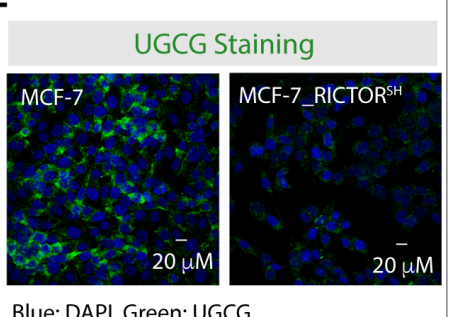

Blue: DAPI, Green: UGCG

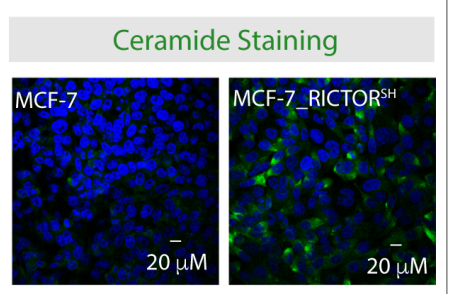

Blue: DAPI, Green: Ceramide $\mathbf{0}$

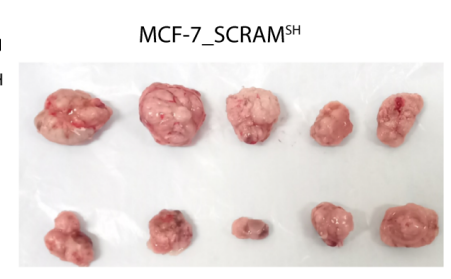

MCF-7_RICTOR ${ }^{\text {SH }}$
P

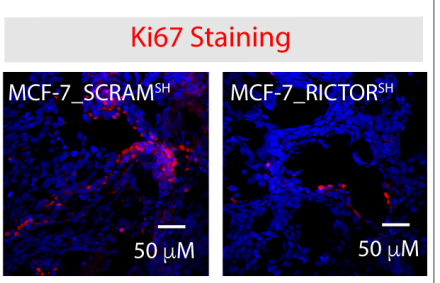

Blue: DAPI, Red: Ki67

RICTOR regulates sphingolipid metabolism via UGCG regulation.

Q

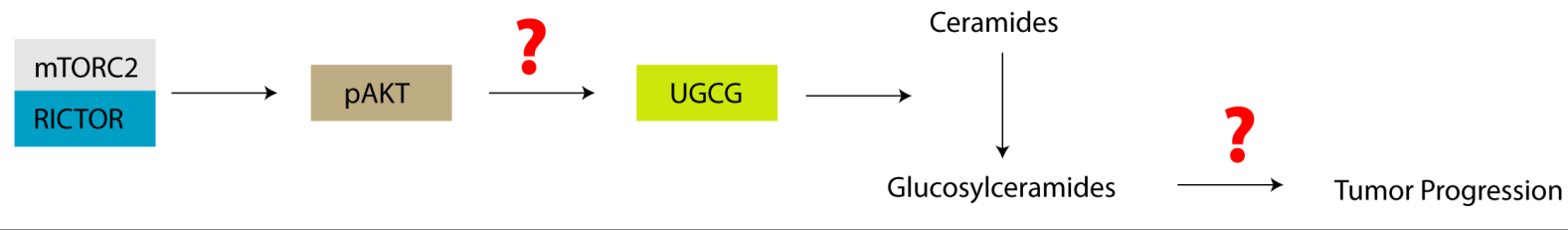


Figure 2. RICTOR Silencing Reduces Glucosylceramide Levels, and Inhibits Tumor Progression.

(A) Immunoblot confirming the knockdown of RICTOR expression in MCF-7_RICTOR ${ }^{\text {SH }}$ cells. (B) Cell proliferation kinetic studies show decrease in proliferation of MCF-7_RICTOR ${ }^{\text {SH }}$ cells (mean $\pm S D, n=4$ ) as compared to MCF-7_SCRAM ${ }^{\mathrm{SH}}$ cells. (C, D) Immunoblots (C) and their quantification (D) showing changes in protein expression (mean $\pm S D, n=4$ ) of RICTOR, RAPTOR, and their downstream effectors. (E) Normalized absolute quantitation (mean, $n=5)$ of ceramide and glucosylceramide species (C16:0, C18:0, C20:0, C22:0, C24:0, and C24:1) in MCF-7 and MCF-7_RICTOR ${ }^{\text {SH }}$ cells. (F, G) Fold change in levels of sphingolipid species reveal an increase in levels of ceramides (F) and decrease in levels of glucosylceramides (G) in MCF-7_RICTOR ${ }^{\text {SH }}$ cells as compared to MCF-7 cells. (H) Immunofluorescence imaging confirms enhanced levels of ceramides in MCF-7_RICTOR ${ }^{\text {SH }}$ cells. (l, J, K) qRT-PCR (mean \pm SD, $\mathrm{n}=3$ ) $(\mathrm{I})$, immunoblots $(\mathrm{J})$, and their quantification (mean $\pm S D, n=3)(K)$ demonstrate downregulation of UGCG without any change in GBA1 expression in MCF-7_RICTOR ${ }^{\text {SH }}$ cells as compared to MCF-7 cells. (L) Immunofluorescence imaging confirms decreased expression of UGCG in MCF-7_RICTOR ${ }^{\text {SH }}$ cells. (MO) Tumor growth kinetics (M), final day tumor volume $(\mathbf{N})$, and pictures of excised tumors (O) show a significantly slower growth of MCF-7_RICTOR ${ }^{S H}$ (mean $\pm S E M, n=5$ ) tumors as compared to MCF7_SRAM ${ }^{\text {SH }}$ tumors. (P) Immunofluorescence images reveal low expression of Ki67 in MCF-7_RICTOR tumor sections as compared to MCF-7_SCRAM ${ }^{\mathrm{SH}}$ tumors. (Q) A schematic diagram showing the role of putative factor/s modulating the RICTOR/pAKT-mediated UGCG expression that can lead to altered levels of glucosylceramides, controling tumor progression. Data in Figure 2D, 2F, 2G, 2I, 2K, and 2N were analysed using an unpaired student's $t$-test, and in Figure 2B and 2M was analysed using Twoway ANOVA. $p$-value: ${ }^{\star} p<0.05,{ }^{* *} p<0.01,{ }^{* *} p<0.001,{ }^{* * * *} p<0.0001$. 


\section{RICTOR Regulates UGCG Expression via Transcription Factor Zinc Finger-X linked (ZFX).}

We argued that if RICTOR silencing in MCF-7 cells downregulates UGCG expression, then this regulation may be mediated through transcription factor/s that gets downregulated upon RICTOR silencing. Therefore, we performed differential gene expression analysis on mouse Rictor (-) microarray datasets from NCBI GEO, and identified the transcription factors that are downregulated on RICTOR knockdown (Figure 3A) (31). Using bioinformatic analysis, we also identified experimentally validated transcription factors that bind to the UGCG promoter in MCF-7 cells (Figure 3A) (32). We found three common transcription factors, Zinc Finger X-linked (ZFX), ETS transcription factor (E-74) Like Factor 1 (ELF1), and CCCTC-binding Factor (CTCF) from above two data sets that may bind to UGCG promoter, and are transcriptionally downregulated on RICTOR silencing. qRT-PCR results confirmed that MCF-7_ RICTOR $^{\text {SH }}$ cells have reduced expression of all these three transcription factors (Figure 3B). We further validated the binding of ZFX on UGCG promoter by ChiP-qPCR, and observed $\sim 1.5$-fold $(p<0.05)$ decrease in recruitment of ZFX on UGCG promoter in MCF-7_RICTOR ${ }^{\text {SH }}$ cells as compared to MCF-7 cells (Figure 3C, Supplementary Figure S3A). In line with this, MCF-7_RICTOR ${ }^{\text {SH }}$ cells showed $\sim 1.5$-fold $(p<0.05)$ decrease in ZFX protein expression as compared to MCF-7 cells (Figure 3D, 3E).

\section{ZFX Regulates RICTOR-mediated UGCG expression.}

ZFX is a highly conserved Zinc figure protein and oncogenic transcription factor residing on the $X$ chromosome, and is overexpressed in many cancers (33-35). To functionally validate the role of ZFX in UGCG regulation, we used ZFX-overexpressed MCF-7 cells (MCF-7_ZFXOE), and only vector overexpressed (MCF-7_VECT ${ }^{\mathrm{OE}}$ ) cells as control, along with siRNA-mediated ZFX-silenced MCF-7 cells $\left(M C F-7 \_Z F X^{S L}\right)$ with scrambled siRNA-transfected cells (MCF-7_SCRAM ${ }^{S L}$ ) as control. qRT-PCR (Supplementary Figure S3B) and immunoblot analysis (Figure 3F, 3G) confirmed the overexpression of ZFX in MCF-7_ZFXOE cells, and downregulation of ZFX in MCF-7_ZFX ${ }^{\mathrm{SL}}$ cells.

Quantitative estimation of ceramides and glucosylceramides by LC-MS/MS showed 1.4-1.8 fold decrease in levels of ceramide species, and 2-8 fold increase in levels of glucosylceramide species in MCF-7_ZFX ${ }^{\mathrm{OE}}$ cells as compared to MCF-7_VECT ${ }^{\mathrm{OE}}$ cells (Figure $3 \mathrm{H}, 3 \mathrm{I}$ ). In contrast, we observed a 1.83.8-fold increase in levels of ceramide species, and a 1.5-3.0-fold decrease in levels of glucosylceramide 
species on ZFX silencing (Figure 3H, 3I) that was further validated by ceramide staining (Figure 3J). These alterations in levels of ceramides and glucosylceramides on ZFX overexpression and silencing prompted us to quantify the UGCG expression. As expected, MCF-7_ZFXOE cells showed $\sim 2$-fold $(p<$ $0.05)$ increase, and MCF-7_ZFXSL cells showed $\sim 2$-fold $(p<0.05)$ downregulation of UGCG expression by qRT-PCR (Supplementary Figure S3C) that was validated by immunoblot (Figure $3 \mathrm{~K}, 3 \mathrm{~L}$ ) and immunofluorescence imaging for UGCG (Figure 3M).

\section{ZFX-mediated UGCG Regulation Controls Tumor Progression.}

To elucidate the effect of ZFX-mediated UGCG regulation on cell proliferation and tumor progression, we compared cell proliferation rates of MCF-7_ZFX ${ }^{\mathrm{OE}}$ and MCF-7_ZFX ${ }^{\mathrm{SL}}$ cells. MCF-7_ZFXOE exhibited a significant increase in cell proliferation as compared to MCF-7_VECTOE cells, and ZFX silencing showed $>1.4$-fold $(p<0.001)$ decrease in proliferation as compared to MCF-7_SCRAM ${ }^{S L}$ cells after $72 \mathrm{~h}$ (Supplementary Figure S3D). Scratch wound assay showed $>30 \%$ increase $(p<0.0001)$ in cell migration in MCF-7_ZFXOE cells, whereas ZFX silencing marked a $120 \%$ decrease $(p<0.0001)$ after $36 \mathrm{~h}$ (Supplementary Figure S3E) that was further validated by transwell migration assay (Supplementary Figure S3F). Animal studies recorded a significantly higher growth rate of MCF-7_ZFXOE tumors as compared to MCF-7_VECT ${ }^{\mathrm{OE}}$ tumors (Figure 3N) that was confirmed by Ki67 staining (Figure 30).

To validate UGCG-mediated enhanced cell proliferation and tumor progression upon ZFX overexpression, we determined cell proliferation rates of MCF-7_ZFXOE cells upon siRNA-mediated UGCG silencing. Proliferation kinetics demonstrated that UGCG silencing causes a decrease in proliferation rate of MCF-7_ZFXOE cells (Figure 3P). Similarly, nanogel-mediated delivery of UGCG siRNA caused $>1.4$-fold $(p<0.0001)$ decrease in tumor growth kinetics of MCF-7_ZFXOE tumors (Figure 3Q). Therefore, these results affirm that ZFX, regulated by RICTOR, is one of the transcription factors that modulate UGCG expression in Luminal representative MCF-7 cells, priming the increase in glucosylceramide levels, and enhancing the tumor progression (Figure 3R). Therefore, the next step was to delineate how RICTOR controls ZFX-mediated UGCG expression (Figure 3R). 
Figure 3

ZFX is the key transcription factor that binds to UGCG promoter

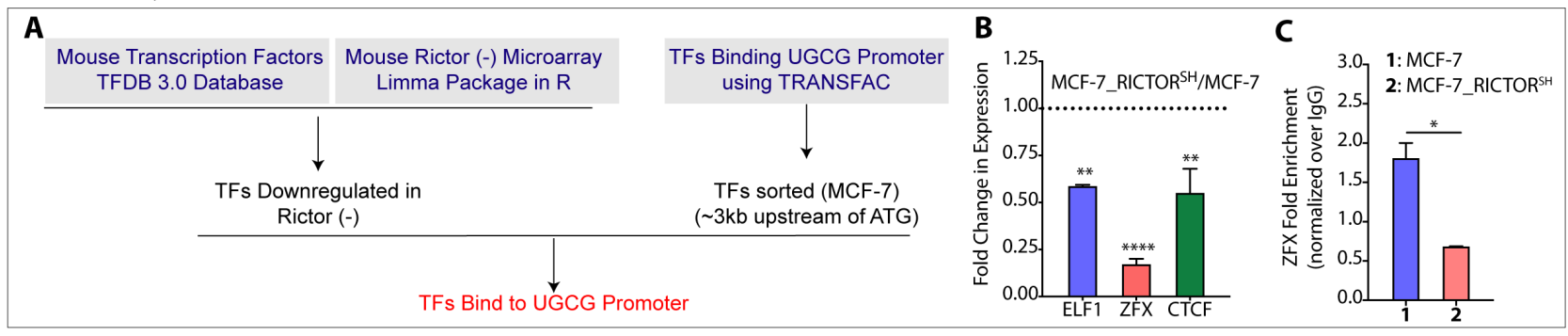

ZFX alters the levels of ceramides and glucosylceramides.

D

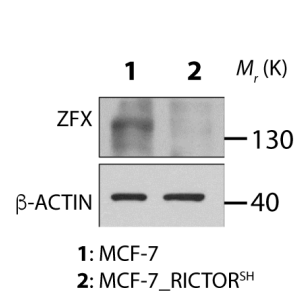

H

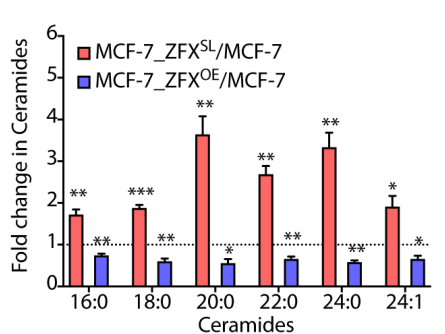

E

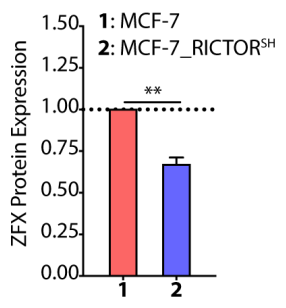

I

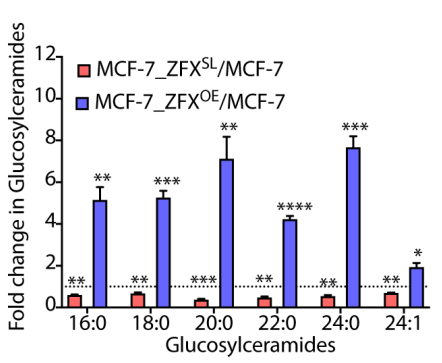

\section{$\mathbf{F}$}

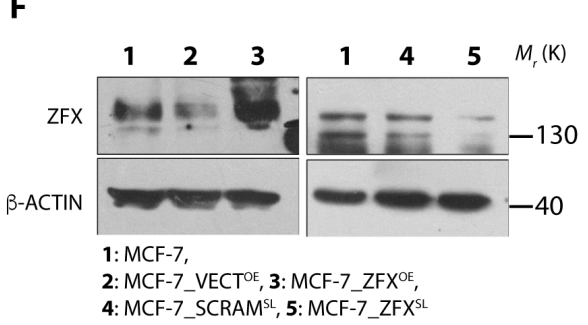

G

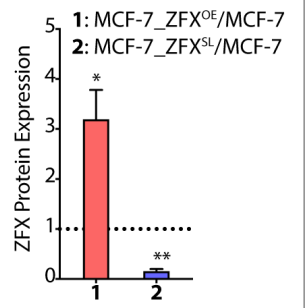

J

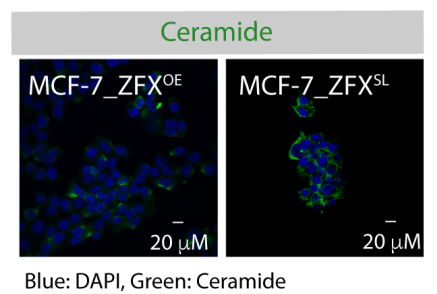

Blue: DAPI, Green: Ceramide

ZFX regulates cell proliferation and tumor progression via UGCG.

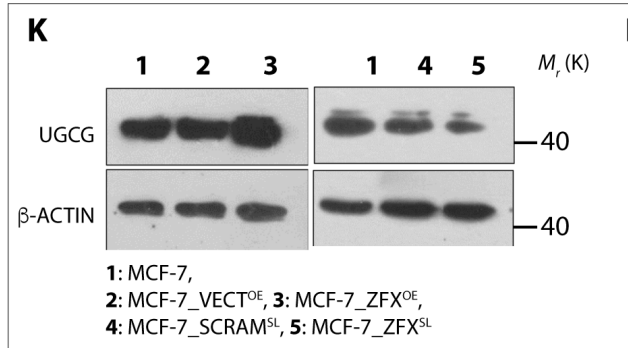

L

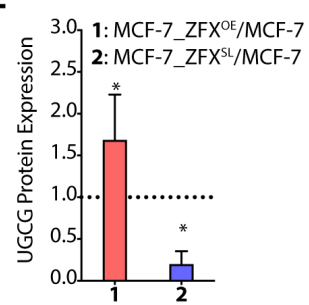

M

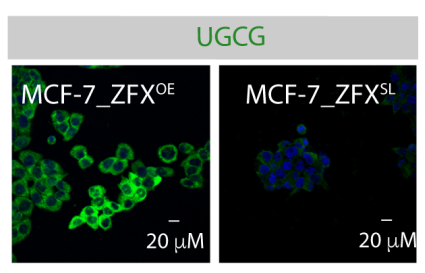

Blue: DAPI, Green: UGCG
$\mathbf{N}$

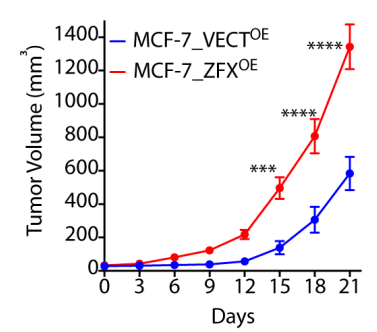

O

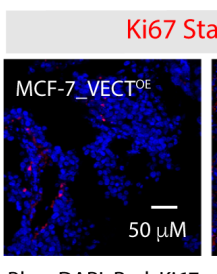

Blue: DAPI, Red: Ki67

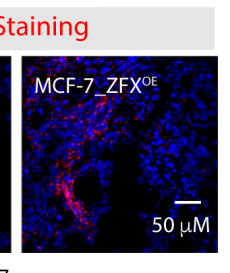

$\mathbf{P}$

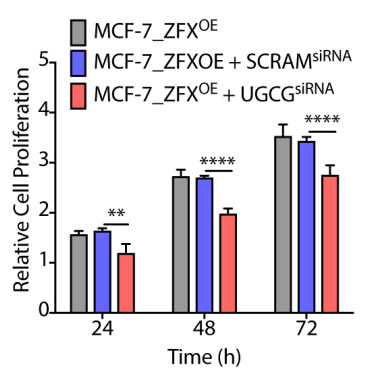

\section{Q}

- MCF-7_ZFXOE

§ $3500 \cdot$ MCF-7_ZFXOE + SCRAM ${ }^{\text {SRNA }}$

3000 - MCF-7_ZFXOE + UGCG GiRNA

छ 3000- MCF-7_ZFXOE + UGCGG

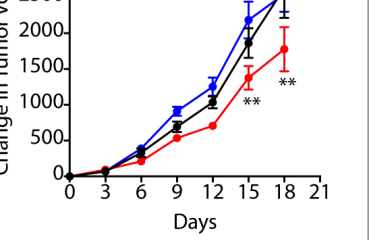

RICTOR regulates UGCG transcription via ZFX.

$\mathbf{R}$
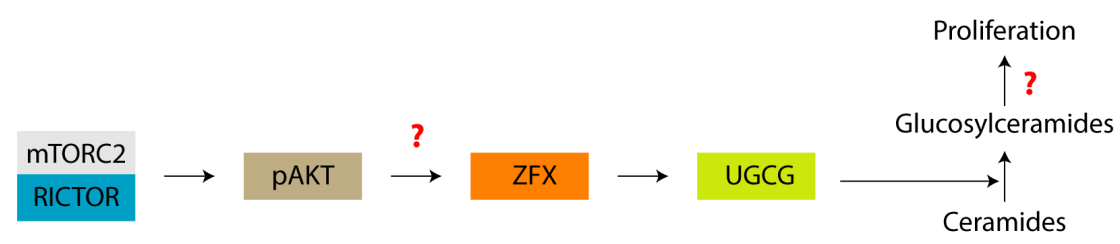
Figure 3. RICTOR Regulates UGCG Expression via Transcription Factor Zinc Finger-X linked (ZFX).

(A) A schematic diagram showing the workflow used for identification of RICTOR-regulated transcription factors that can bind to UGCG promoter. (B) qRT-PCR confirm reduced expression of RICTOR-regulated ELF1, ZFX, and CTCF transcription factors in MCF-7_RICTOR ${ }^{\text {SH }}$ cells. (C) ChIP-qPCR (mean $\pm S D, n=3$ ) results show reduced binding of ZFX to the UGCG promoter in MCF-7_RICTOR cells. (D, E) Immunoblots (D) and their quantification (mean $\pm S D, n=3$ ) $(E)$ show downregulation of ZFX expression in MCF-7_RICTOR ${ }^{\mathrm{SH}}$ cells. $(\mathrm{F}, \mathrm{G})$ Immunoblots $(\mathrm{F})$ and their quantification (mean $\pm S D, \mathrm{n}$ $=3)(G)$ confirm overexpression and silencing of ZFX in MCF-7_ZFXOE and MCF-7_ZFX ${ }^{S L}$ cells respectively. $(H, I)$ Fold change (mean \pm SEM, $n=5$ ) in absolute levels of ceramides $(H)$ and glucosylceramides (I) confirm reduced levels of ceramides and higher levels of glucosylceramides in MCF-7_ZFXOE cells. In contrast, MCF-7_ZFX ${ }^{S L}$ cells show higher levels of ceramides and reduced levels of glucosylceramides. (J) Immunofluorescence staining support low levels of ceramides in MCF-7_ZFXOE cells, and high ceramide levels in MCF-7_ZFXSL cells. $(K, L)$ Immunoblots $(K)$ and their quantification (mean $\pm \mathrm{SD}, \mathrm{n}=3$ ) (L) witness upregulation and downregulation of UGCG upon overexpression and silencing of ZFX in MCF-7_ZFXOE and MCF-7_ZFX ${ }^{S L}$ cells respectively. (M) Immunofluorescence staining support high UGCG expression in MCF-7_ZFXOE cells, and lower expression in MCF-7_ZFXSL cells. (N) Tumor growth kinetics recorded a significantly higher growth of MCF-7_ZFXOE (mean \pm SD, $n=4$ ) tumors as compared to MCF-7_VECT ${ }^{\mathrm{OE}}$ tumors. (O) Immunofluorescence images reveal increased expression of Ki67 in MCF-7_ZFXOE tumor sections as compared to MCF-7_VECTOE tumor sections. (P, Q) siRNA-mediated silencing of UGCG causes a significant decrease in proliferation of MCF-7_ZFXOE cells (P) and reduced tumor growth kinetics in MCF-7_ZFXOE tumors (Q). (R) A schematic diagram showing the role of regulatory mechanism/s in modulating the RICTOR/pAKT-mediated regulation of ZFX/UGCG that leads to altered levels of glucosylceramides and controls tumor progression. Data in Figure $3 \mathrm{~B}, 3 \mathrm{C}, 3 \mathrm{E}, 3 \mathrm{G}-\mathrm{I}$, and $3 \mathrm{~L}$ were analysed using an unpaired student's $t$-test, and in Figure $3 \mathrm{~N}, 3 \mathrm{P}$, and 3Q were analysed using Two-way ANOVA. $p$-value: ${ }^{\star} p<0.05,{ }^{* *} p<0.01,{ }^{* \star *} p<0.001,{ }^{* \star *} p<$ 0.0001 . 


\section{RICTOR Regulates UGCG Expression via Epigenomic Alterations.}

pAKT-mediated regulation of DNA methyltransferases (DNMTs), histone demethylases (HDMs), and histone methyltransferases (HMTs) can lead to epigenomic alterations, and influence the gene transcription (36). UGCG promoter has multiple CpG islands, and ZFX is known to bind at CpG islands in gene promoters (Supplementary Figure S4) (37). Therefore, we hypothesized that pAKT-mediated regulation of DNMTs may interfere with binding of ZFX to the UGCG promoter (38) (Figure 4A). We, first checked the DNMT expression in MCF-7_RICTOR ${ }^{S H}$ cells, and found high expression of DNMT1, DNMT3A, and DNMT3B in comparison to MCF-7 cells (Figure 4B). This enhanced expression may be responsible for increased DNA methylation, and reduced UGCG expression in MCF-7_RICTOR ${ }^{\text {SH }}$ cells.

To validate DNMT-mediated UGCG regulation, we inhibited the DNMTs by decitabine (DAC) treatment $(2,5,10 \mu \mathrm{M})$, and found 2-4-fold $(p<0.05)$ increase in UGCG expression by qRT-PCR (Figure 4C). Immunoblot studies confirmed the increase in UGCG expression in MCF-7_ RICTOR ${ }^{\text {SH }}$ cells on DAC treatment (Figure 4D). Further experiments were done with an optimum $5 \mu \mathrm{M}$ DAC concentration. Using ChIP-qPCR, we witnessed a 2-fold $(p<0.05)$ increase in ZFX enrichment and binding to UGCG promoter in DAC-treated MCF-7_RICTOR ${ }^{S H}$ cells (Figure 4E). Therefore, DAC (DNMT inhibition)mediated upregulation of UGCG is due to enhanced recruitment of ZFX at the UGCG promoter site. We further confirmed the increase in UGCG expression (Figure 4F) and decrease in ceramides (Figure 4G) by immunofluorescence staining in MCF-7_RICTOR ${ }^{S H}$ cells on DAC treatment. DAC treated MCF7_RICTOR ${ }^{\mathrm{SH}}$ cells also showed increased cell proliferation (Figure 4H) and cell migration (Figure 4I). Therefore, these studies demonstrate that DAC-mediated downregulation of DNMTs in MCF7_RICTOR ${ }^{S H}$ cells enhanced the recruitment of ZFX, and elevated UGCG expression, due to reduced DNMT-mediated methylation of UGCG CpG islands. 


\section{RICTOR Regulates UGCG Expression via Histone Demethylase KDM5A.}

Promoter-associated trimethylation of histone $\mathrm{H} 3(\mathrm{H} 3 \mathrm{~K} 4 \mathrm{Me} 3)$ is one of the key targets of PI3K/AKT that acts as a mode of regulating transcriptional competence (39). H3K4Me3 status is regulated by histone demethylase, KDM5A (40). AKT-mediated phosphorylation of KDM5A is instrumental in its nuclear exit leading to elevated H3K4Me3 levels, thereby augmenting gene transcription (Figure 4J). Inhibition of KDM5A using a chemical inhibitor, KDOAM-25, demonstrated an increase in UGCG gene expression by 1.5-2.0 fold ( $<$ 0.05) (Figure 4K), that was validated by immunoblot studies showing an increase in UGCG expression with concurrent decrease in KDM5A expression (Figure 4L). ChIP-PCR results confirmed that MCF-7_RICTOR ${ }^{\text {SH }}$ cells with reduced AKT activation possess a reduced H3K4Me3 mark as compared to MCF-7 cells, and it was reverted upon KDM5A inhibition (30 $\mu \mathrm{M})$ with a $>1.3$-fold $(p=$ 0.057) increase (Figure 4M). Immunofluorescence imaging further confirmed the increase in UGCG expression (Figure $4 \mathrm{~N}$ ).

As KDM5A inhibition causes an increase in UGCG expression and decreases ceramides, cellular assays confirmed an increased cell proliferation (Figure 4O) and cell migration in MCF-7_ RICTOR ${ }^{\mathrm{SH}}$ cells (Figure 4P) on KDM5A inhibition. We further validated the effect of KDM5A inhibiton on UGCG regulation and tumor progression in NOD SCID mice where KDM5A inhibiton enhances the tumor growth kinetics of MCF-7_ RICTOR ${ }^{S H}$ cells (Figure 4Q). Therefore, these results confirm that mTORC2AKT mediated phosphorylation of KDM5A does not allow the demethylation of H3K4Me3, and enhanced H3K4Me3 activates UGCG transcription, leading to tumor progression. To complete the circuit connecting the metabolic-gene regulatory signalling, the next step was to find how UGCGmediated increase in glucosylceramides is responsible for enhanced tumor progression (Figure 4R). 
Figure 4

DNMTs regulate ZFX-mediated UGCG transcription in MCF-7 cells.

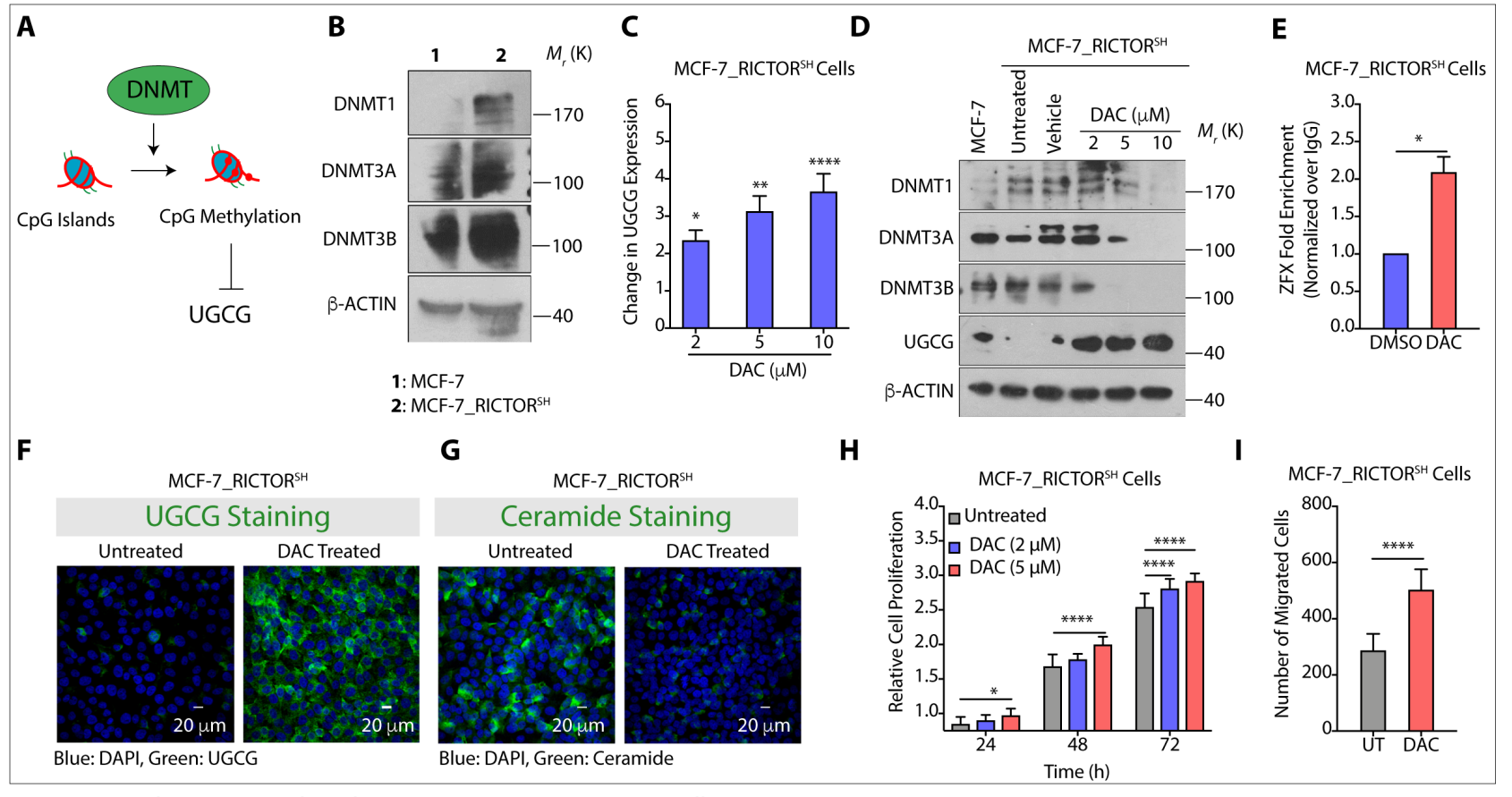

KDM5A regulates ZFX-mediated UGCG transcription in MCF-7 cells.

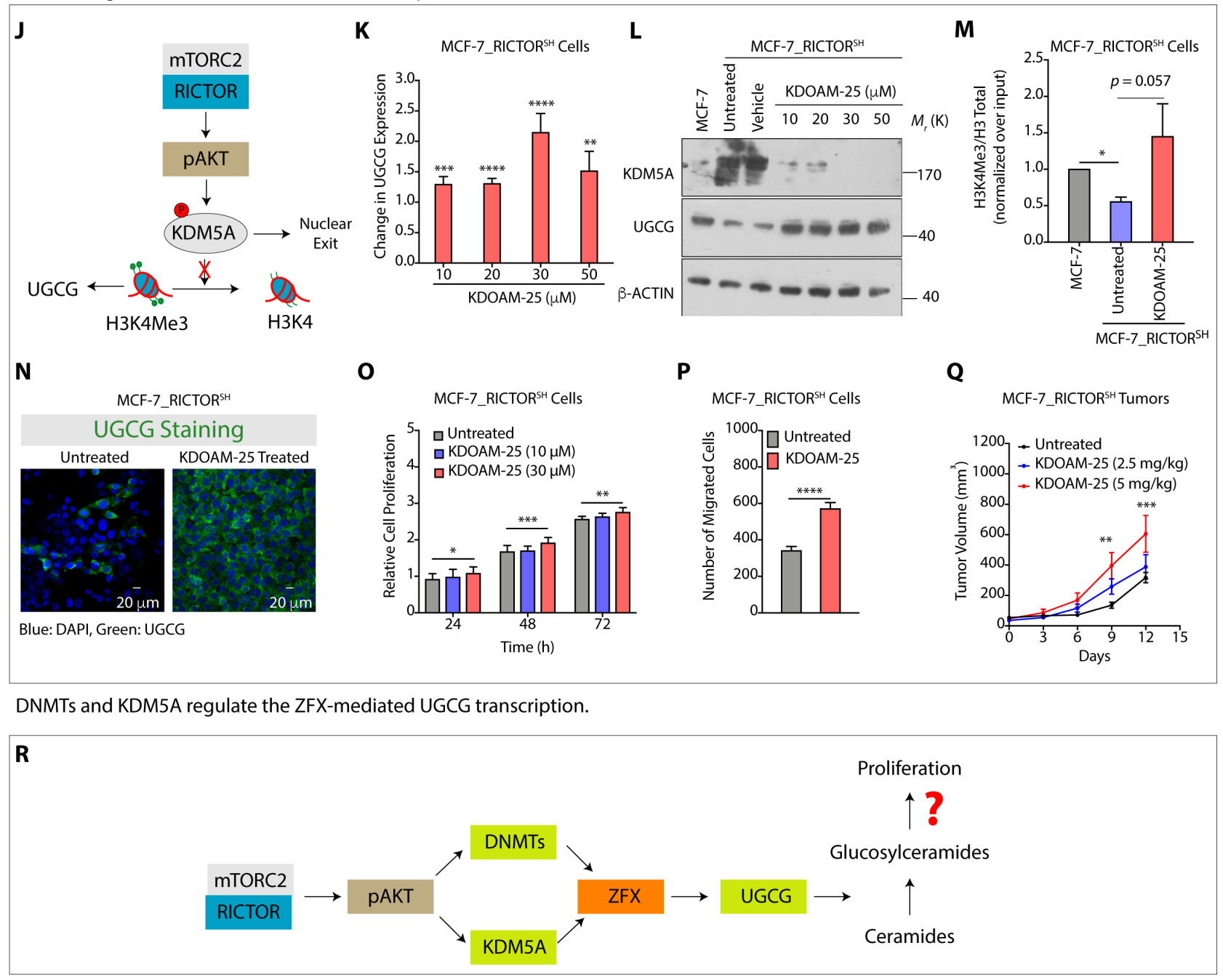




\section{Figure 4. AKT Regulates UGCG Expression via Epigenomic Alterations.}

(A) A schematic diagram showing DNMT-mediated methylation of CpG islands that further regulates the transcriptional competence of UGCG. (B) Immunoblots showing an increase in expression of DNMTs in MCF-7_RICTOR ${ }^{S H}$ cells as compared to MCF-7 cells. (C) qRT-PCR results show an increase in expression of UGCG on inhibition of DNMTs by DAC inhibitor. (D) Immunoblots reveal a decrease in expression of DNMTs with a concurrent increase in UGCG expression in MCF-7_RICTOR ${ }^{\text {SH }}$ cells upon DAC treatment. (E) ChIP-qPCR results (mean $\pm S D, n=3$ ) confirm enhanced binding of ZFX to UGCG promoter in MCF-7_RICTOR ${ }^{S H}$ cells on DAC $(5 \mu \mathrm{M})$ treatment. $(F, G)$ Immunofluorescence images confirm enhanced expression of UGCG (F) and decreased levels of ceramides (G) in MCF-7_RICTOR ${ }^{\text {SH }}$ cells on DAC $(5 \mu \mathrm{M})$ treatment. $(H, I)$ Cell proliferation (mean $\pm S D, n=3)(H)$ and cell migration (mean $\pm S D, n=3)(I)$ assays confirm increased proliferation rate and migration of MCF-7_RICTOR ${ }^{S H}$ cells on DAC $(5 \mu \mathrm{M})$ treatment. (J) A schematic representation of pAKT-mediated regulation of histone demethylase KDM5A, and its ability to regulate UGCG transcription via histone methylation (H3K4Me3). $(K)$ qRT-PCR (mean $\pm S D, n=3$ ) results demonstrate an increase in UGCG expression in MCF-7_RICTOR ${ }^{S H}$ cells upon treatment with KDM5A chemical inhibitor, KDOAM-25. (L) Immunoblot showing an increase in UGCG expression and decrease in KDM5A expression after treatment of MCF-7_RICTOR ${ }^{\text {SH }}$ cells with KDOAM-25 inhibitor. (M) ChIP-qPCR results (mean $\pm S D, n=3$ ) show reduced H3K4me3 mark on UGCG promoter in MCF-7_RICTOR ${ }^{S H}$ cells that gets enhanced on treatment with KDOAM-25 inhibitor (30 $\left.\mu \mathrm{M}\right)$. (N) Immunofluorescence images confirm enhanced expression of UGCG in MCF-7_RICTOR ${ }^{\text {SH }}$ cells on KDM5A inhibition $(30 \mu \mathrm{M}) .(\mathrm{O}, \mathrm{P})$ Cell proliferation (mean $\pm \mathrm{SD}, \mathrm{n}=3)(\mathrm{O})$ and cell migration (mean \pm $S D, n=3)(P)$ assays confirm increased proliferation and migration of MCF-7_RICTOR ${ }^{\text {SH }}$ cells on KDM5A inhibition. (Q) Inhibition of KDM5A causes a significant increase in tumor growth kinetics of MCF7_RICTOR ${ }^{\text {SH }}$ cells. (R) A schematic diagram showing pAKT substrates DNMTs and KDM5A epigenetically regulates ZFX enrichment on UGCG promoter and UGCG transcription leading to altered levels of glucosylceramides and enhanced tumor cell proliferation. Data in Figure 4C, 4E, 4I, 4K, 4M, and 4P were determined using an unpaired student's $t$-test, and in Figure 4H, 4O, and 4Q were analysed using Twoway ANOVA. $p$-value: ${ }^{\star} p<0.05,{ }^{* *} p<0.01,{ }^{* *} p<0.001,{ }^{* * * *} p<0.0001$. 


\section{ZFX-mediated UGCG Regulation Alters the Level of Gangliosides.}

Synthesis of glucosylceramides is followed by synthesis of lactosylceramides and monosialic acid containing GM3 gangliosides (Figure 5A). GM3 gangliosides can either be converted to monosialic acid gangliosides, GM2 and GM1, or disialic acid gangliosides GD3 (Figure 5A). As RICTOR controls ZFXmediated transcriptional regulation of UGCG and synthesis of glucosylceramides, we quantified the impact of ZFX-UGCG regulation on levels of lactosylceramides and three key gangliosides, GM3, GD3, and GM1 in MCF-7, MCF-7_RICTOR ${ }^{\mathrm{SH}}$, MCF-7_UGCG ${ }^{\mathrm{OE}}$, and MCF-ZFX ${ }^{\mathrm{OE}}$ cells. As RICTOR silencing reduces the levels of glucosylceramides, we observed $>4$-fold $(p<0.005)$ decrease in C16:0 lactosylceramides in MCF-7_RICTOR ${ }^{\mathrm{SH}}$ cells as compared to MCF-7 cells (Figure 5B). In contrast, overexpression of ZFX and UGCG induced a 4-8 fold increase in lactosylceramides (Figure 5B). MCF7_RICTOR ${ }^{\text {SH }}$ cells have significantly reduced levels of all species of GM3 gangliosides (C16:0, C18:0, C20:0, C22:0, and C24:0) that may be due to reduced levels of glucosylceramides (Supplementary Figure S5A, Figure 5C). In contrast, there was a several-fold increase in levels of all species of GM3 gangliosides in MCF-7_UGCGOE and MCF-ZFX' ${ }^{\mathrm{OE}}$ cells due to higher expression of glucosylceramides (Supplementary Figure S5A). To reaffirm that UGCG is responsible for these changes in level of gangliosides, we evaluated the expression of other ganglioside-metabolic enzymes (ST8SIA-V, B4GALNT1, B3GALT4, ST8SIA1), and observed no appreciable change in MCF-ZFXOE cells over MCF-7 cells (Supplementary Figure S5B).

As C16:0 GM3 gangliosides are most abundant in MCF-7 cells, and it showed a sharp increase in MCF7_UGCGOE and MCF-7_ZFX ${ }^{\mathrm{OE}}$ cells (Figure 5C, Supplementary Figure S5A), we compared the changes in levels of C16:0-derived GM1 and GD3 gangliosides among different cell types. MCF-7_RICTOR ${ }^{\mathrm{SH}}$ cells showed $>3$-fold $(p<0.05)$ decrease in levels of GM1 gangliosides, and 2.5 - fold $(p<0.05)$ decrease in levels of GD3 gangliosides as compared to MCF-7 cells (Figure 5D, 5E). This reduction in GM1 and GD3 gangliosides on RICTOR silencing is due to reduced levels of glucosylceramides and GM3 gangliosides. In contrast, there was 4-10-fold decrease in GM1 gangliosides, and 1.5-2.0 fold increase in GD3 gangliosides in MCF-7_UGCGOE and MCF-7_ZFX ${ }^{O E}$ cells over MCF-7 cells (Figure 5D, 5E). Interestingly, MCF-7_RICTOR ${ }^{\text {SH }}$ cells have >2-fold higher levels of GM1 gangliosides, and >6-fold lower levels of GD3 gangliosides than MCF-7_UGCGOE and MCF-7_ZFX' cells (Figure 5D, 5E). These alterations in GD3 gangliosides were then validated by flow cytometry using anti-GD3 ganglioside 
antibody (Figure 5F, 5G).

\section{GD3 Gangliosides cause EGFR-mediated Tumor Progression.}

Gangliosides present in GEMs are well-known to regulate RTK signalling that is contingent upon nature and relative quantity of gangliosides, kind of growth factor receptors, and cell type (47). Gangliosides like GD2 and GD3 can activate RTK signalling, and enhance cancer cell proliferation (42). In contrast, gangliosides like GM1 can mitigate RTK signalling via inhibiting the dimerization of growth factor receptors (43). To study the regulatory effects of alterations in gangliosides on RTK signalling, we estimated the levels of phosphorylated epidermal growth factor receptor (EGFR) and its downstream signalling components. Immunoblot analysis showed no alterations in levels of total EGFR in MCF7_RICTOR ${ }^{\mathrm{SH}}$, MCF-7_UGCGOE, and MCF-ZFX ${ }^{\mathrm{OE}}$ cells as compared to MCF-7 cells (Figure 5H). However, we observed $\mathrm{a}>3$-fold $(p<0.05)$ increase in phosphorylated EGFR $\left(\mathrm{EGFR}^{\mathrm{Y} 1173}\right.$ and $\left.\mathrm{EGFR}^{\mathrm{Y} 1068}\right)$ in MCF7_UGCGOE and MCF-7_ZFXOE cells having higher levels of GD3 gangliosides over that of MCF-7 cells (Figure 5I). Similarly, we observed activation and upregulation of downstream signalling intermediates, $\mathrm{PAKT}^{\mathrm{S473}}$, and extracellular signal-regulated protein kinase/p(ERK1/2) in MCF-7_UGCGOE and MCF7_ZFXOE cells in comparison to MCF-7 cells (Figure 5H, 5I). Therefore, these results suggest that overexpression of ZFX or UGCG causes increased activation of EGFR-mediated RTK signalling.

To validate GD3 ganglioside-mediated activation of EGFR, we performed siRNA-mediated silencing of GD3 synthase (ST8SIA1) in MCF-7_ZFXOE cells, and observed a significant downregulation in the pEGFR $^{\mathrm{Y} 1173}$ and $\mathrm{pEGFR}{ }^{\mathrm{Y} 1068}$ levels, though the total EGFR level was unaltered (Figure 5J, 5K). ST8SIA1 silencing using siRNA also attenuated the cell proliferation of MCF-7_ZFX ${ }^{O E}$ cells (Figure 5L), and even abrogated the tumor growth kinetics in mice xenografts (Figure 5M) that was validated by Ki67 staining (Figure $5 \mathrm{~N}$ ). Therefore, these results confirm that GD3-mediated activation of EGFR is responsible for cell proliferation and tumor progression, thus completing the metabolic-signalling-gene regulation circuit. (Figure 50). 
bioRxiv preprint doi: https://doi.org/10.1101/2022.01.10.475595; this version posted January 12, 2022. The copyright holder for this

Figure 5

RICTOR-ZFX-UGCG axis alters ganglioside levels in MCF-7 cells.

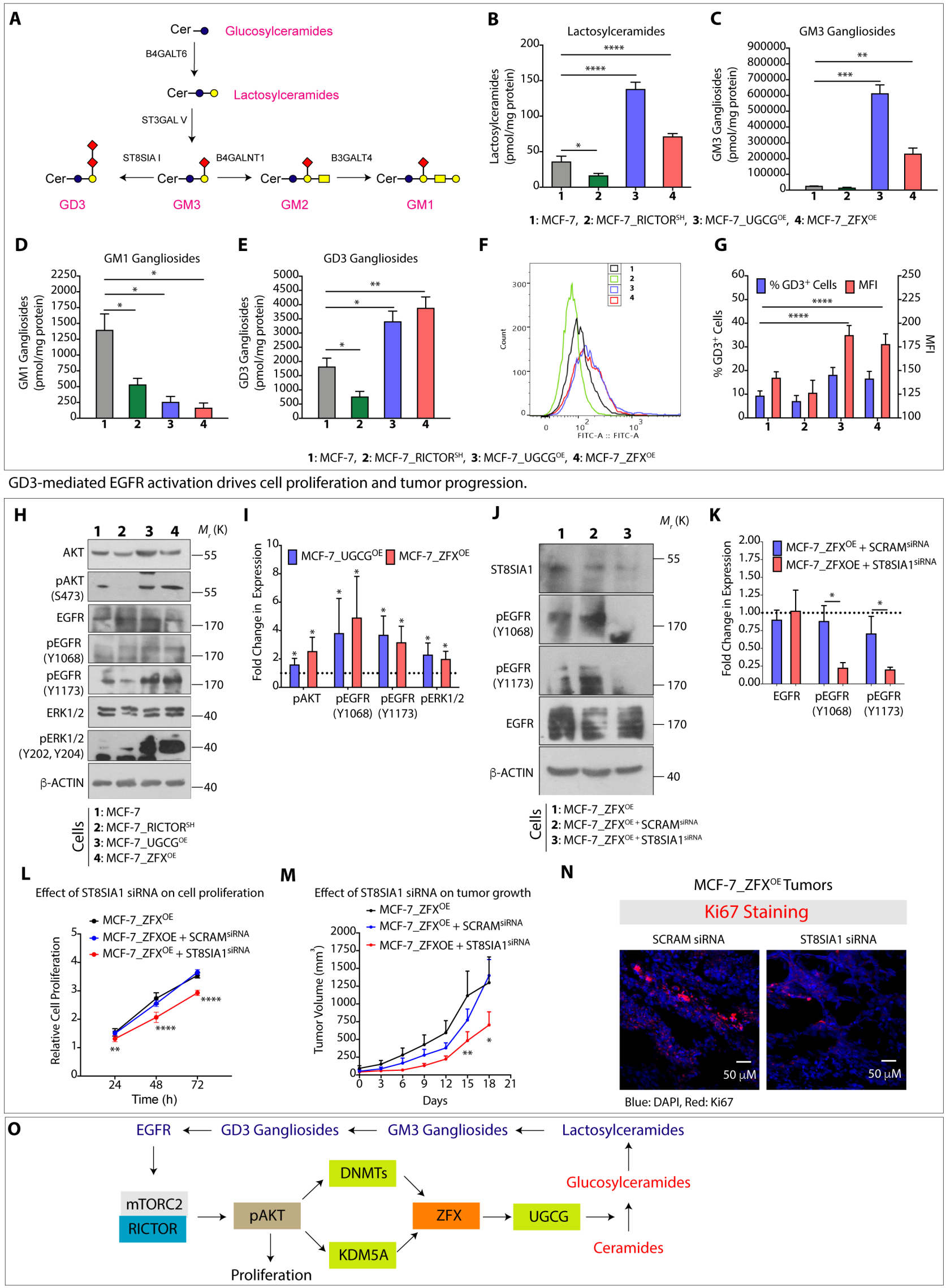


Figure 5. ZFX-mediated UGCG Regulation Alters Levels of Gangliosides and Activates EGFR.

(A) Schematic presentation of a part of ganglioside metabolic pathway showing the synthesis of GD3 and GM2/GM1 gangliosides from common precursor GM3 gangliosides. (B-E) Absolute quantitation (mean \pm SEM, $n=3$ ) of C16:0-derived lactosylceramides (B), GM3 (C), GM1 (D), and GD3 (E) gangliosides show increased levels of GM3 and GD3 gangliosides, and decreased levels of GM1 gangliosides in MCF7_UGCGOE and MCF-7_ZFXOE cells. (F, G) Representative flow cytometry (F) and quantification of GD3 gangliosides (G) using anti-GD3 antibody confirm increased levels in MCF-7_UGCGOE and MCF-7_ZFX ${ }^{O E}$ cells. $(H, I)$ Immunoblots $(H)$ and their quantification (mean $\pm S D, n=3)(I)$ reveal an increase in levels of pEGFR ${ }^{\mathrm{Y} 1173}$, pEGFR ${ }^{\mathrm{Y} 1068}$, $\mathrm{pAKT}^{\mathrm{S473}}$, and pERK1/2 $2^{\left(\mathrm{Y} 202, \mathrm{Y}^{204)}\right.}$ in MCF-7_UGCG ${ }^{\mathrm{OE}}$ and MCF-7_ZFX ${ }^{\mathrm{OE}}$ cells as compared to MCF-7 cells. $(\mathrm{J}, \mathrm{K})$ Immunoblots $(\mathrm{J})$ and their quantification (mean $\pm \mathrm{SD}, \mathrm{n}=3$ ) $(\mathrm{K})$ show decreased activation of EGFR on siRNA-mediated silencing of ST8SIA1. (L) Cell proliferation assay demonstrates decrease in cell proliferation (mean $\pm S D, n=3$ ) of MCF-7_ZFXOE cells on siRNA-mediated inhibition of GD3 synthase (ST8SIA1). (M, N) Tumor growth kinetics using Xenograft studies (M) and Ki67 stained images $(\mathrm{N})$ show a decrease in growth kinetics (mean \pm SEM, $n=5-6$ ) of MCF-7_ZFXOE tumors on siRNA-mediated inhibition of GD3 synthase (ST8SIA1). (O) A schematic diagram showing the role of ZFX-mediated transcriptional regulation of UGCG that regulates ganglioside biosynthesis and ganglioside-mediated EGFR activation. Data in Figure 5-E, 5G, 5I, and 5K were analysed using an unpaired student's $t$-test, and in Figure $5 \mathrm{~L}$ and 5M was analysed using Two-way ANOVA. $p$-value: * $p<$ $0.05,{ }^{* *} p<0.01,{ }^{* *} p<0.001,{ }^{* * * *} p<0.0001$. 


\section{ZFX Expression is Strongly Associated with UGCG in Luminal Patients.}

To find the association between ZFX and UGCG in Luminal breast cancer patients, we analyzed the TCGA-BRCA and METABRIC patient datasets. TCGA patient dataset $(\mathrm{N}=1082)$ based on PAM50 classification ( $N=833$ ) was divided into Luminal $A(N=416)$, Luminal $B(N=185)$, and non-Luminal subtypes $(N=232)$ (Figure $6 A)(44)$. Differential gene expression data analysis showed that Luminal A and B tumor tissues have significantly higher expression of UGCG (Figure 6B) and ZFX (Figure 6C) as compared to Basal and HER2 ${ }^{+}$groups. Correlating ZFX and UGCG expression to ER and PR status revealed that $\mathrm{ER}^{+}$(Figure 6D, 6E) and $\mathrm{PR}^{+}$(Figure 6F, 6G) tumors have significantly higher expression of UGCG (Figure 6D, 6F) and ZFX (Figure 6E, 6G). Gene expression analysis further revealed that $>56 \%$ of Luminal (Luminal A and B) patients have high expression of both UGCG and ZFX (Figure 6H). Similarly, we divided the METABRIC data sets based on PAM50 classification $(N=1905)$ into Luminal $A(N=696)$, Luminal B ( $N=474)$, and non-Luminal ( $=728)$ subtypes (Supplementary Figure S6A) (45). Metadata analysis further confirmed that Luminal $A$ and Luminal B tumor tissues have higher UGCG and ZFX expression over other subtypes as observed in TCGA data set (Supplementary Figure S6B, S6C), and expression of UGCG and ZFX is also high in $\mathrm{ER}^{+}$and $\mathrm{PR}^{+}$tumor tissues (Supplementary Figure S6D-G). We identified $45 \%$ of Luminal (Luminal A and B) patients with higher UGCG expression also exhibit higher ZFX expression (Supplementary Figure S6H).

To further validate the association of ZFX and UGCG in breast cancer patients, we quantified the expression of UGCG and ZFX by immunohistochemical (IHC) analysis in tumor samples of all subtypes $(\mathrm{N}=90)$ (Dataset 2). In concurrence with TCGA BRCA and METABRIC datasets, IHC analysis confirmed that $\sim 15 \%$ of Luminal patients have high ZFX and UGCG expression (Figure $6 \mathbf{l}$, J). Finally, we quantified the expression of UGCG and ZFX from Luminal patients by qRT-PCR, and observed $\sim 2$-fold $(p<0.05)$ increase in expression of ZFX and UGCG in tumor tissues over adjacent matched normal control (Figure $6 \mathrm{~K})$. Therefore, these results support a positive correlation between ZFX and UGCG expression in Luminal patients. 
Figure 6

Association of high UGCG and ZFX expression in Luminal tumors from TCGA dataset.

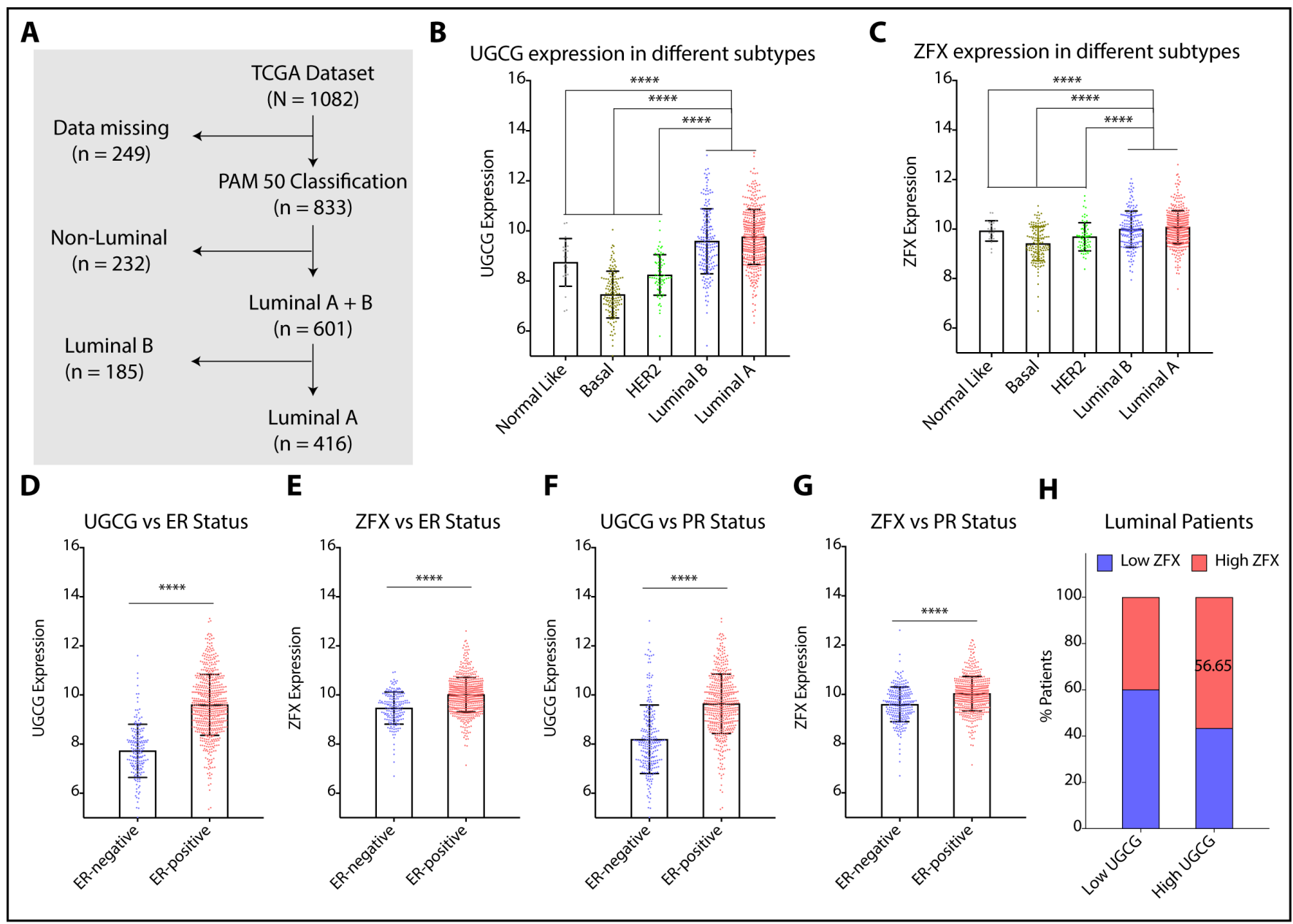

Association of UGCG and ZFX expression in Luminal tumors from Indian cohort.

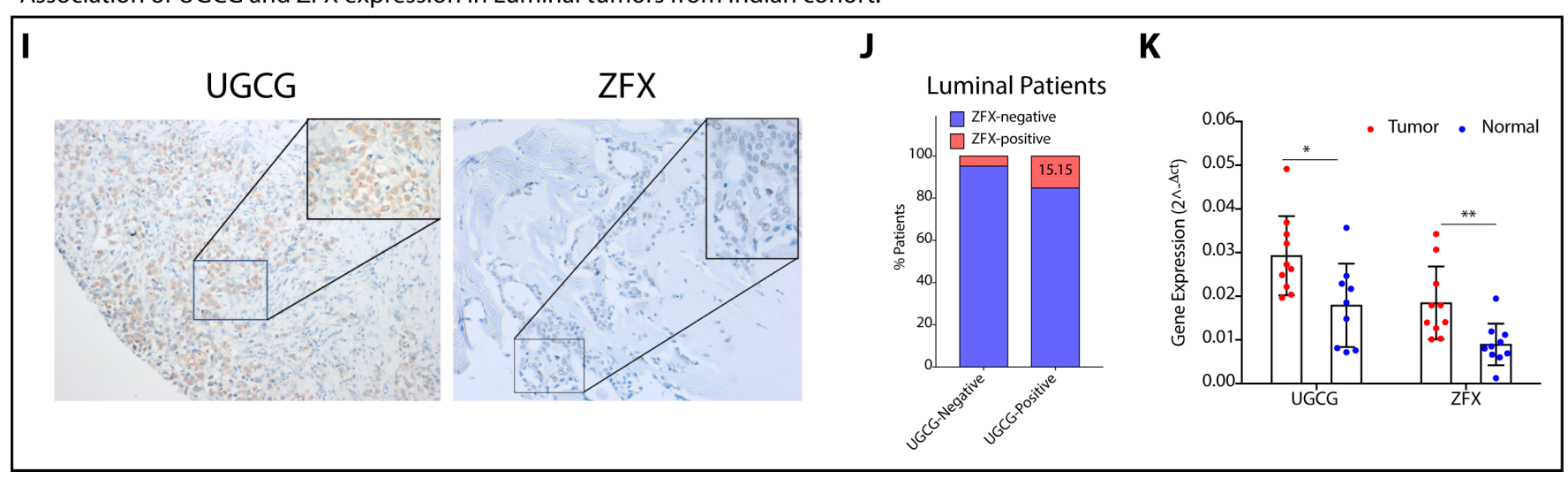


Figure 6. ZFX Expression is Strongly Associated with UGCG in Luminal Patients.

(A) A schematic diagram showing the PAM50 classification of TCGA tumor dataset used for analysis. (B,

C) Gene expression of UGCG (B) and ZFX (C) in different breast cancer subtypes (PAM50) of TCGA dataset confirms high expression of UGCG and ZFXin Luminal subtypes as compared to other subtypes. (D-G) Change in expression of $\operatorname{UGCG}(\mathrm{D}, \mathrm{F})$ and $\operatorname{ZFX}(\mathrm{E}, \mathrm{G})$ with respect to $E R(\mathrm{D}, \mathrm{E})$ and $P R(F, G)$ status in breast tumors of TCGA dataset confirm high $\operatorname{UGCG}(\mathrm{D}, \mathrm{F})$ and high $Z F X(\mathrm{E}, \mathrm{G})$ expression in $\mathrm{ER}^{+}$and $\mathrm{PR}^{+}$tumors. (H) Percentage of tumors having high expression of UGCG and ZFXamong Luminal subtype tumors in TCGA data set. (I) Representative immunohistochemical images show enhanced cytoplasmic UGCG and increased nuclear stain of ZFX in Luminal breast tissues. All images are at 100X magnification, and insets are at 400x magnification. (J) Percentage of Luminal tumors from Indian cohort $(\mathrm{N}=90)$ positive for both UGCG and ZFX on immunohistochemical staining. (K) qRT-PCR (mean $\pm S D, n=10$ ) validation showing high UGCG and high ZFX expression from Luminal subtype tumors in comparison to adjacent normal tissues in an Indian cohort. Data in Figure S6B and S6C were analyzed using Oneway ANOVA, and data in Figure S6D-S6G and S6K were analyzed by an unpaired student's $t$-test. $p$ value: ${ }^{*} p<0.05,{ }^{* *} p<0.01,{ }^{* * *} p<0.0001$. 


\section{DISCUSSION}

Treating cancer is like the Herculean duel with the chthonic creature Hydra, whose decapitation magically led to a botanical duplication of the regenerated heads. The myriad of alternative strategies that cancer cells employ to achieve survival advantage over the clinical interventions is a similar saga. One of the prime reason for this is our lack of complete understanding of the metabolic signaling and gene regulatory networks that cancer cells deploy in order to survive. More important is to understand how these networks are interconnected so that multiple nodes in the circuit/network can be combinatorially targeted to overrule their survival strategies. In this context, herein, we have mapped the first step of metabolic-signaling-gene regulatory circuit connecting ganglioside metabolism with cancer, controlled by EGFR-mTORC2/RICTOR complex (Figure 7).

The major biological roles of sphingolipids and gangliosides at the cell surface include modulating the lipid phase of cellular membranes, acting as ligands for membrane receptors, kinases, and enzymes, and surface recognition through glycan interactions by glycosphingolipids (46). Gangliosides, as a part of GEMs, can act as double-edged swords where they can either augment or inhibit the growth factormediated cell proliferation through activation/deactivation of RTK signalling cascades (47). Using an elegant precedence of ganglioside-mediated activation of growth factor receptor signalling, our work reveals that increased GD3 and GM3 levels and reduced GM1 levels in response to altered expression of UGCG boost the EGFR autophosphorylation status, and subsequent downstream growth signalling in Luminal cancers. Although RTK inhibitors have made breakthroughs in tumor treatment options, however, RTK co-activation networks mark a serious limitation in their use (48). This effectively suggests that systematic effort in manipulation of gangliosides via UGCG or GD3/GM3 synthesizing enzymes can serve as a strategy to prevent activation of multiple RTKs that network for accelerated tumor growth in Luminal subtype.

Targeting RICTOR downstream of RTKs may be another node that can be tapped simultaneously. We ruled out the cross-talk of mTORC1 on RICTOR silencing, as RAPTOR and downstream targets like pS6 Kinase and p4EBP1 were unchanged in MCF-7_RICTORSH cells. However, there are many negative feedback loops working between the mTOR cascades; which though important, investigating all of these were beyond the scope of this study. Although many studies till now have reported high UGCG expression in ER-positive Luminal tumors $(49,50)$, but none delved into its molecular mechanism. Our 
study on the other hand unravelled and validated that, ZFX, a key C2H2-type, ZNF family transcription factor mediates UGCG expression, and thereby modulates the sphingolipid and ganglioside metabolism, and Luminal tumor progression. Thus, to avoid the activation of mTOR-mediated negative feedback loops promoting cell proliferation, more effort may be diverted on manipulation of ZFX controlled UGCG expression that will mimic the effect of RICTOR inhibition without the associated side effects. This regulatory effect of ZFX may be one of the reasons why genetic manipulations to silence ZFX in breast, colorectal, pancreatic and renal cancers reduced proliferation and invasion, and predicted a good prognosis $(51,52)$. It also needs to be emphasized that depending on the cell line and growth signalling pathway involved, there may be multiple transcription factor/s regulating the UGCG in a context-dependent manner.

PI3K/AKT signalling stabilizes DNA methyltransferases affecting the global methylation pattern and transcriptional activation of genes in different cancers (53). Transcription factor mediated maintenance of DNMTs to promoters involve co-operative association or recruitment of additional co-repressors (54). In presence of multiple CpG islands in UGCG promoter, transcription factor mediated switch of its activator and repressor effect probably shifts the balance of DNMT/ZFX ratio more towards binding of DNMTs (DNMT1, 3A and 3B) on this oncogene promoter that compromises ZFX binding in MCF7_RICTOR ${ }^{\text {SH }}$ cells. However, it remains to be seen which of the DNMTs, if not more than one member, are recruited to UGCG promoter that prevents ZFX enrichment when PAKT levels are low. Similarly, AKT-mediated phosphorylation of KDM5A causes it nuclear export, and allows the ZFX binding to the promoter by allowing histone methylation. Besides, pAKT-mediated epigenetic regulations can be further exploited to control gene expression mediated metabolic networking. In summary, our study endorses that EGFR-mTORC2-RICTOR-AKT-UGCG-Ganglioside circuit regulates tumor progression in Luminal breast cancer cells, and provides us an opportunity to intervene at multiples nodes to tame cancer cells. It is prudent to mention that the underlined paradigm of mTORC2/RICTOR regulated expression of UGCG impacting the level of glucosylceramides may be part of a fundamental mechanism in breast tumor development especially in Luminal tumors as shown in clinical samples, that certainly requires more attention and research. 


\section{Cancer cell}

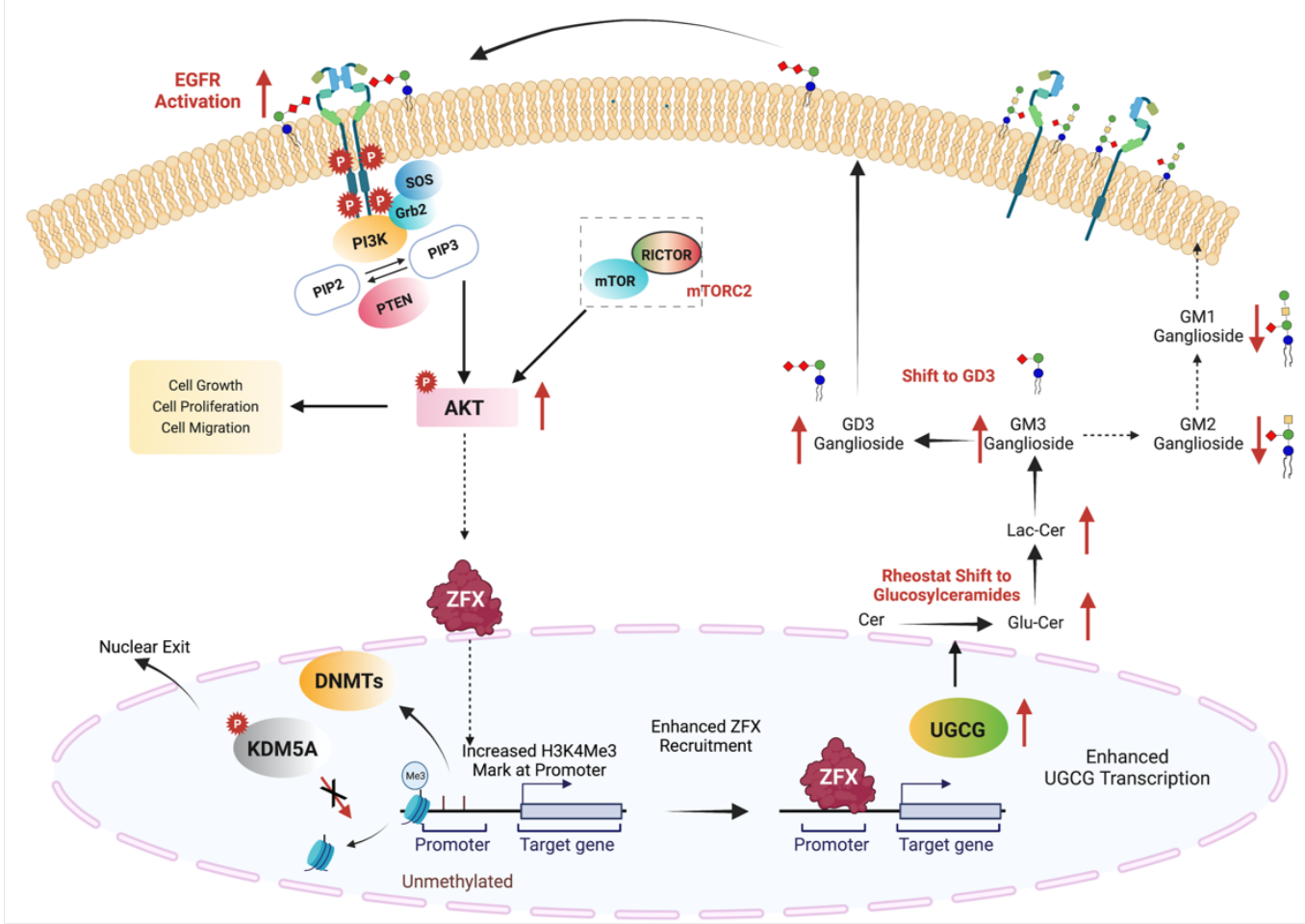

\section{RICTOR Knockdown in cancer cell}

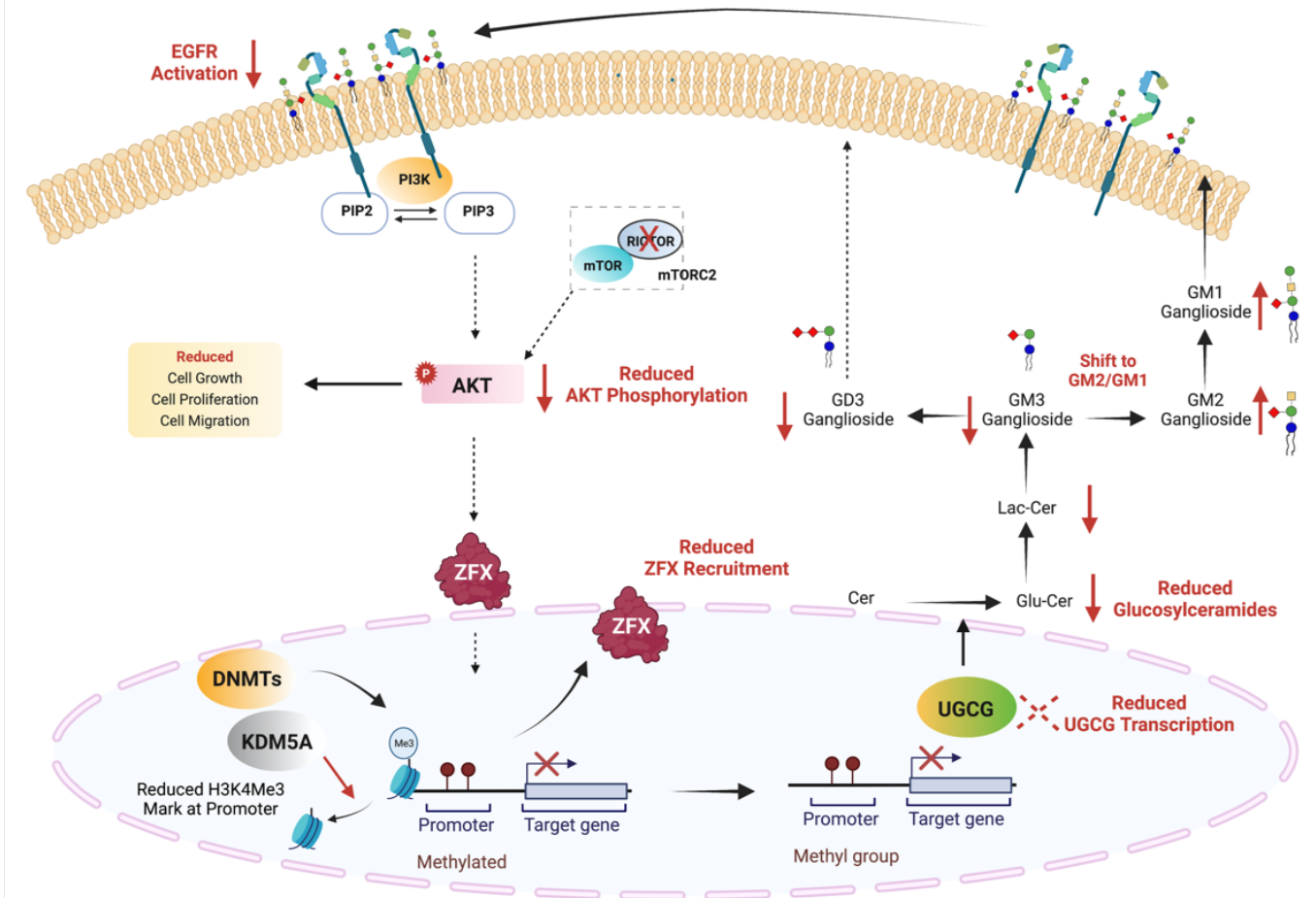

Figure 7. Schematic showing the metabolic-signaling-gene regulatory circuit connecting ganglioside metabolism with cancer. Ganglioside-mediated EGFR activation followed by mTORC2/RICTOR signalling epigenetically modifies UGCG transcription efficiency, ultimately leading to tumor progression. 


\section{ACKNOWLEDGEMENTS}

We are grateful to Dr. Sagar Sengupta, Dr. Vinay Nandicoori, Dr. Kaustav Bandopadhyay, and Dr. Tapas Mukherjee for many helpful discussions. pERK1/2 antibody was kind gift from Dr. Vinay Nandicoori. We thank DST-FIST sponsored Amity Lipidomics Research Facility at Amity University Haryana. We thank biorepository of Rajiv Gandhi Cancer Institute and Research Centre (RGCIRC), Delhi. Figure 7 was drawn using BioRender.com with publication licence numbers OZ23FCUP9X and PA23FCUPHN.

\section{FUNDING}

The support from Amity University Haryana, RCB, AIIMs, and Department of Biotechnology (DBT) Govt. of India is greatly acknowledged. Research in U.D. group is supported by BT/PR19624/BIC/101/488/2016 (DBT)， BT/PR40413/BRB/10/1922/2020 (DBT), CRG/2021/002966 (SERB), and 5/13/81/2020/NCDIII (ICMR). Research in AB group is supported by BT/PR40413/BRB/10/1922/2020 (DBT). AM is supported by SERB-STAR award (STR/2019/000064) and DBT-National Bioscience Award for Career Development (BT/HRD/NBA/38/04/2016). Amity Lipidomics Research Facility at Amity University Haryana is supported by DST-FIST grant, SR/FST/LSI-664/2016. A.K. thanks ICMR, and S.J. and K.Rana thank CSIR for research fellowships. We are grateful to Nadathur Estates for their support of all the breast cancer research activities at SJRI. We acknowledge the support of the DBT e-Library Consortium (DeLCON) for providing access to e-resources. Animal work in the small animal facility of Regional Centre for Biotechnology is supported by BT/PR5480/INF/22/158/2012 (DBT).

\section{ETHICAL STATEMENT.}

All animal experiments were performed after due approval of the Institutional Animal Ethical Committee of Regional Centre for Biotechnology (RCB/IAEC/2020/079) as per the guidelines of Committee for the Purpose of Control and Supervision of Experiments on Animals (CPCSEA), India. All studies with human tissue samples were conducted after due ethical clearance from AllMS (IEC-332/01.07.2016), RGCIRC (RGCIRC/IRB/276/2019, Res/BR/TRB-20/2020/ 70), Amity University Haryana (IEC-AIISH/AUH/ 2016-1), and SJRTI ( S475/79-80).

\section{AUTHOR CONTRIBUTIONS}

K.R. performed all cell culture experiments and immunofluorescence studies. M.N.A. performed and analysed all gene and protein expression studies and ChIP analysis. S.K.J. and K.Rajput performed the 
animal studies. N.M., P.S., K.C., and A.K. performed sphingolipid and ganglioside estimation experiments. S.D performed the bioinformatic analysis. K.Rana performed flow cytometry experiments. A.K. created shRNA silenced cell lines, and performed activity assays. T.P., G.M, and J.P. compiled patient clinical information. S.Deo provided Luminal patient tissue samples, and patient clinical information. J.P. performed the immunohistochemistry analysis and metadata analysis. A.M. supervised bioinformatic analysis and validation. K.R., M.N.A., A.K. compiled the data. All authors read the manuscript and gave their input. U.D. conceived the idea. U.D. and A.B. wrote the manuscript, and supervised the whole project.

\section{SUPPORTING INFORMATION.}

Complete list of supporting figures, tables, and data sets are in supporting information.

\section{REFERENCES}

1. Y. A. Hannun, and L. M. Obeid, Sphingolipids and their Metabolism in Physiology and Disease. Nat. Rev. Mol. Cell. Biol. 19, 175-191 (2018).

2. X. Zhang, and F. L. Kiechle, Glycosphingolipids in Health and Disease. Ann. Clin. Lab. Sci. 34, 3-13 (2004).

3. B. Ogretmen, Sphingolipid Metabolism in Cancer Signalling and Therapy. Nat. Rev. Cancer 18, 33-50 (2018).

4. S. Sonnino, L. Mauri, V. Chigorno, and A. Prinetti, Gangliosides as Components of Lipid Membrane Domains. Glycobiol. 17, 1R-13R (2007)

5. S. Julien, M. Bobowski, A. Steenackers, X. L. Bourhis, and P. Delannoy, How Do Gangliosides Regulate RTKs Signaling? Cells 2, 751-767 (2013).

6. G. Hoxhaj, and B. D. Manning, The PI3K-AKT Network at the Interface of Oncogenic Signalling and Cancer Metabolism. Nat. Rev. Cancer 20, 74-88 (2020).

7. E. Paplomata, and R. O'Regan, The PI3K/AKT/mTOR Pathway in Breast Cancer: Targets, Trials and Biomarkers. Ther. Adv. Med. Oncol. 6, 154-166 (2014)

8. L. C. Kim, R. S. Cook, and J. Chen, mTORC1 and mTORC2 in Cancer and the Tumor Development. Oncogene 36, 2191-2201 (2017).

9. E. Jacinto, R. Loewith, A. Schmidt, S. Lin, M. A. Rüegg, A. Hall, and M. N. Hall, Mammalian TOR Complex 2 Controls the Actin Cytoskeleton and is Rapamycin Insensitive. Nat. Cell Biol. 6, 1122-1128 (2004). 
10. D. D. Sarbassov, S. M. Ali, S. Sengupta, J. Sheen, P. P. Hsu, A. F. Bagley, A. L. Markhard, and D. M. Sabatini, Prolonged Rapamycin Treatment Inhibits mTORC2 Assembly and Akt/PKB. Mol. Cell. 22, 159168 (2006).

11. J. M. García-Martínez, and D. R. Alessim, mTOR complex 2 (mTORC2) Controls Hydrophobic Motif Phosphorylation and Activation of Serum- and Glucocorticoid-Induced Protein Kinase 1 (SGK1). Biochem. J. 416, 375-385 (2008).

12. F. Zhang, X. Zhang, M. Li, P. Chen, B. Zhang, H. Guo, W. Cao, X. Wei, X. Cao, X. Hao, and N. Zhang, mTOR Complex Component Rictor Interacts with PKCd and Regulates Cancer Cell Metastasis. Cancer Res. 70, 9360-9370 (2010).

13. H. Cheng, Y. Zou, J. S. Ross, K. Wang, X. Liu, B. Halmos, S. M. Ali, H. Liu, A. Verma, C. Montagna, A. Chachoua, S. Goel, E. L. Schwartz, C. Zhu, J. Shan, Y. Yu, K. Gritsman, R. Yelensky, D. Lipson, G. Otto, M. Hawryluk, P. J. Stephens, V. A. Miller, B. Piperdi, and R. Perez-Soler, RICTOR Amplification Defines A Novel Subset of Patients with Lung Cancer who may Benefit from Treatment with mTORC1/2 Inhibitors. Cancer Discov. 5, 1262-1270 (2015).

14. S. T. Kim, S. Y. Kim, S. J. Klempner, J. Yoon, N. Kim, S. Ahn, H. Bang, K. M. Kim, W. Park, S. H. Park, J. O. Park, Y. S. Park, H. Y. Lim, S. H. Lee, K. Park, W. K. Kang, and J. Lee, Rapamycin-Insensitive Companion of mTOR (RICTOR) Amplification Defines A Subset of Advanced Gastric Cancer and is Sensitive To AZD2014-mediated mTORC1/2 inhibition. Ann. Oncol. 28, 547-554 (2017).

15. M. M. Joly, D. J. Hicks, B. Jones, V. Sanchez, M. V. Estrada, C. Young, M. Williams, B. N. Rexer, D. D. Sarbassov, W. J. Muller, D. Brantley-Sieders, and R. S. Cook, Rictor/mTORC2 Drives Progression and Therapeutic Resistance of HER2-Amplified Breast Cancers. Cancer Res. 76, 4752-4764 (2016).

16. L. Wang, J. Qi, J. Yu, H. Chen, Z. Zou, X. Lin, and L. Guo, Overexpression of Rictor Protein in Colorectal Cancer is Correlated with Tumor Progression and prognosis. Oncol. Lett. 14, 6198-6202 (2017).

17. H. Bang, S. Ahn, E. J. Kim, S. T. Kim, H. Y. Park, J. Lee, and K. Kim, Correlation between RICTOR Overexpression and Amplification in Advanced Solid Tumors. Pathol. Res. Pract. 216, 152734 (2020).

18. J. Gao, B. A. Aksoy, U. Dogrusoz, G. Dresdner, B. Gross, S. O. Sumer, Y. Sun, A. Jacobsen, R. Sinha, E. Lasson, E. Cerami, C. Sander, and N. Schultz, Integrative Analysis of Complex Cancer Genomics and Clinical Profiles using cBioPortal. Sci. Signal. 6, 11 (2013)

19. K. Mashui, W. K. Cavenee, and P. S. Mischel, mTORC2 in the Center of Cancer Metabolic 
Reprogramming. Trends Encroinol. Metab. 25, 364-373 (2014).

20. D. W. Lamming, and D. M. Sabatini, A Central Role for mTOR in Lipid Homeostasis. Cell Metab. 18, 465-469 (2013).

21. D. Berchtold, M. Piccolis, N. Chiaruttini, I. Riezman, H. Riezman, A. Roux, T. C. Walther, and R. Loewith, Plasma Membrane Stress Induces Relocalization of SIm Proteins and Activation of TORC2 to Promote Sphingolipid Synthesis. Nat. Cell. Biol. 14, 542-547 (2012).

22. B. J. Niles, A. C. Joslin, T. Fresques, and T. Powers, TOR Complex 2-Ypl1 Signaling Maintains Sphingolipid Homeostasis by Sensing and Regulating ROS Accumulation. Cell Rep. 6, 541-552 (2014).

23. Y. Guri, M. Colombi, E. Dazert, S. K. Hindupur, J. Roszik, S. Moes, P. Jenoe, M. H. Heim, I. Riezman, H. Riezman, and M. N. Hall, mTORC2 Promotes tumorigenesis via Lipid Synthesis. Cancer Ce// 32, 807823 (2017).

24. M. Ignatiadis, and C. Sotiriou, Luminal Breast Cancer: From Biology to Treatment. Nat. Rev. Clin. Oncol. 10, 494-506 (2013).

25. O. Yersal, and S. Barutca, Biological Subtypes of Breast Cancer: Prognostic and Therapeutic Implications. World. J. Clin. Oncol. 5, 412-424 (2014).

26. N. Medatwal, and U. Dasgupta. 2020. Quantitation of Sphingolipids in Mammalian Cell Lines by Liquid Chromatography-Mass Spectrometry. In: Prasad R., Singh A. (eds) Analysis of Membrane Lipids. Springer Protocols Handbooks: 103-117. Springer, New York, NY. (Book Chapter)

27. S. Pal, N. Medatwal, S. Kumar, A. Kar, V. Komalla, P. S. Yavvari, D. K. Mishra, D. Malakar, M. Pillai, A. Srivastava, R. D. Sharma, S. Sengupta, U. Dasgupta, and A. Bajaj, A Localized Chimeric Hydrogel Therapy Targets Tumor Microenvironment through Alteration of Sphingolipid Metabolism. ACS Central Sci. 5, 1648-1662 (2019).

28. M. R. Paul, T.i Pan, D. K. Pant, N. N.C. Shih, Y. Chen, K. L. Harvey, A. Solomon, D. Lieberman, J. J. D. Morrissette, D. Soucier-Ernst, N. G. Goodman, S. W. Stavropoulos, K. N. Maxwell, C. Clark, G. K. Belka, M. Feldman, A. DeMichele, and L. A. Chodosh, Genomic Landscape of Metastatic Breast Cancer Identifies Preferentially Dysregulated Pathways and Targets. J. Clin. Invest. 130, 4252-4265 (2020).

29. S. Cha, E. Lee, and H. Won, Comprehensive Characterization of Distinct Genetic Alterations in Metastais Breast Cancer across Various Metastatic Sites. Nph Breast Cancer7, Article number 93 (2021). 30. A. Jebali, and N. Dumaz, The Role of RICTOR Downstream of Receptor Tyrosine Kinase in Cancers. 
Mol. Cancer 17, 39 (2018).

31. H.Hu, Y. Miao, Q. Yu, Q. Zhang, and A. Guo, Animal TFDB 3.0: A Comprehensive Resource for Annotation and Prediction of Animal Transcription Factors. Nucleic Acids Res. 47, D33-D38 (2019).

32. M. E. Ritchie, B. Phipson, D. Wu, Y. Hu, C. W. Law, W. Shi, and G. K. Smyth, limma Powers Differential Expression Analyses for RNA-sequencing and Microarray Studies. Nucleic Acids Res. 43, e47 (2015).

33. K. P. Lai, J. Chen, M. He, A. K.K. Ching, C. Lau, P. B.S. Lai, K. To, and N. Wong, Overexpression of ZFX Confers Self-renewal and Chemoresistance Properties in Hepatocellular Carcinoma. Int. J. Cancer 135, 1790-1799 (2014)

34. J. Jiang, and L. Liu, Zinc Finger Protein X-linked is Overexpressed in Colorectal Cancer and is Associated with Poor Prognosis. Oncol. Lett. 10, 810-814 (2015).

35. M. Ganji-Arjenaki, M. Emadi-Baygi, H. Teimori, M. Moradi-Chaleshtori, and V. Mehrzad, ZFX Overexpression in Breast Cancer Positively Correlates with Metastasis. Res. Mol. Med. 4, 45-49 (2016).

36. J. M. Spangle, T. M. Roberts, and J. J. Zhao, The Emerging Role of PI3K/AKT-mediated Epigenetic Regulation in Cancer. Biochim. Biophys. Acta. Rev. Cancer 1868, 123-131 (2017).

37. S. K. Rhie, L. Yao, Z. Luo, H. Witt, S. Schreiner, Y Guo, A. A. Perez, and P. J. Farnham, ZFX Acts as a Transcriptional Activator in Multiple Types of Human Tumors by Binding Downstream from Transcription Start Sites at the Majority of CpG Island Promoters. Genome Res. 28, 310-320 (2018).

38. G. Micevic, V. Muthusamy, W. Damsky, N. Theodosakis, X. Liu, K. Meeth, E. Wingrove, M. Santhanakrishnan, and M. Bosenberg, DNMT3b Modulates Melanoma Growth by Controlling Levels of mTORC2 Component RICTOR. Cell Rep. 14, 2180-2192 (2016).

39. J. M. Spangle, K. M. Dreijerink, A. C. Groner, H. Cheng, C. E. Ohlson, J. Reyes, C. Y. Lin, J. Bradner, J. J. Zhao, T. M. Roberts, and M. Brown, PI3K/AKT Signaling Regulates H3K4 Methylation in Breast Cancer. Cell Rep. 15, 2692-2704 (2016).

40. K. M. Harmeyer, N. D. Facompre, M. Herlyn, and D. Basu, JARID1 Histone Demethylase: Emerging Targets in Cancer. Trends Cancer 3, 713-725 (2017).

41. S. Groux-Degroote, M. Rodríguez-Walker, J. H. Dewald, J. L. Daniotti, and P. Delannoy, Gangliosides in Cancer Cell Signaling. Prog. Mol. Biol. Transl. Sci. 156, 197-227 (2018).

42. R. Li, J. Manela, Y. Kong, and S. Ladisch, Cellular Gangliosides Promote Growth Factor-induced Proliferation of Fibroblasts. J. Biol. Chem. 275, 34213-34223 (2000). 
43. M. Nishio, S. Fukumoto, K. Furukawa, A. Ichimura, H. Miyazaki, S. Kusunoki, T. Urano, and K. Furukawa, Overexpressed GM1 Suppresses Nerve Growth Factor (NGF) Signals by Modulating the Intracellular Localization of NGF Receptors and Membrane Fluidity in PC12 cells. J. Biol. Chem. 279, 33368-33378 (2004).

44. The Cancer Genome Atlas Network. Comprehensive Molecular Portraits of Human Breast Tumors. Nature 490, 61-70 (2012).

45. C. Curtis, S. P. Shah, S. F. Chin, G. Turashvili, O. M. Rueda, M. J. Dunning, D. Speed, A. G. Lynch, S. Samarajiwa, Y. Yuan, S. Gräf, G. Ha, G. Haffari, A. Bashashati, R. Russell, S. McKinney, S. Aparicio, J. D. Brenton, I. Ellis, D. Huntsman, S. Pinder, L. Murphy, H. Bardwell, Z. Ding, L. Jones, B. Liu, I. Papatheodorou, S. J. Sammut, G. Wishart, S. Chia, K. Gelmon, C. Speers, P. Watson, R. Blamey, A. Green, D. MacMillan, E. Rakha, C. Gillett, A. Grigoriadis, E. de Rinaldis, A. Tutt, M. Parisien, S. Troup, D. Chan, C. Fielding, A. T. Maia, S. McGuire, M. Osborne, S. M. Sayalero, I. Spiteri, J. Hadfield, L. Bell, K. Chow, N. Gale, M. Kovalik, Y. Ng, L. Prentice, S. Tavaré, F. Markowetz, A. Langerød, E. Provenzano, A. Purushotham, A. L. BørresenDale and C. Caldas, The Genomic and Transcriptomic Architecture of 2,000 Breast Tumours Reveals Novel Subgroups. Nature 486, 346-352 (2012).

46. G. A. Patwardhan, and Y. Y. Liu, Sphingolipids and Expression Regulation of Genes in Cancer. Prog. Lipid Res. 50, 104-114 (2014).

47. K. Kaucic, Y. Liu, and S. Ladisch, Modulation of Growth Factor Signaling by Gangliosides: Positive or Negative? Methods Enzymol. 417, 168-185 (2006).

48. D. Zhao, M. Jiang, X. Zhang, and H. Hou, The Role of RICTOR Amplification in Targeted Therapy and Drug Resistance. Mol. Med. 26 Article number 20 (2020).

49. J. Liu, P. Sun, Y. Sun, A. Liu, D. You, F. Jiang, and Y. Sun, Expression of Glucosylceramide Synthase in Invasive Ductal Breast Cancer may be Correlated with High Estrogen Receptor Status and Low HER-2 Status. Diagn. Pathol. 9, Article number 22 (2014).

50. E. Puckhaberle, T. Karn, L. Hanker, R. Gatje,m D. Metzler, U. Holtrich, M. Kaufmann, and A. Rody, Prognostic Relevance of Glucosylceramide Synthase (GCS) Expression in Breast Cancer. J. Cancer Res. Clin. Oncol. 135, 81-90 (2009).

51. J. Jen, and Y. C. Wang, Zinc Finger Proteins in Cancer Progression. J. Biomed. Sci. 23, 1-9 (2016). 
52. S. K. Rhie, L. Yao, Z. Luo, H. Witt, S. Schreiner, Y. Guo, A. A. Perez,and P. J. Farnham, ZFX Acts as a Transcriptional Activator in Multiple Types of Human Tumors by Binding Downstream from Transcription Start Sites at The Majority of CpG Island Promoters. Genome Res. 28, 310-320 (2018).

53. T. Zuo, T. M. Liu, X. Lan, Y. I. Weng, R. Shen, F. Gu, Y. W. Huang, S. Liyanarachchi, D. E. Deatherage, P. Y. Hsu, C. Taslim, B. Ramaswamy, C. L. Shapiro, H. J. L. Lin, A. S. L. Cheng, V. X. Jin, and T. H. M. Huang, Epigenetic Silencing Mediated through Activated PI3K/AKT Signaling in Breast Cancer. Cancer Res. 71, 1752-1762 (2011).

54. E. Hervouet, P. Peixoto, R. Delage-Mourroux, M. Boyer-Guittaut, and P Francois, Specific or not Specific Recruitment of DNMTs for DNA Methylation, an Epigenetic Dilemma. Clinical Epigenetics 10, Article number 17 (2018). 